\title{
Comparative Study of View Update Algorithms in Rational Choice Theory *
}

\author{
Radhakrishnan Delhibabu \\ Informatik 5, Knowledge-Based Systems Group \\ RWTH Aachen, Germany \\ delhibabu@kbsg.rwth-aachen.de
}

\begin{abstract}
The dynamics of belief and knowledge is one of the major components of any autonomous system that should be able to incorporate new pieces of information. We show that knowledge base dynamics has interesting connection with kernel change via hitting set and abduction. The approach extends and integrates standard techniques for efficient query answering and integrity checking. The generation of hitting set is carried out through a hyper tableaux calculus and magic set that is focused on the goal of minimality. Many different view update algorithms have been proposed in the literature to address this problem. The present paper provides a comparative study of view update algorithms in rational approach.
\end{abstract}

Keyword: AGM, Belief Revision, Knowledge Base Dynamics, Kernel Change, Abduction, Hyber Tableaux, Magic Set, View update, Update Propagation.

\section{Introduction}

Modeling intelligent agents' reasoning requires designing knowledge bases for the purpose of performing symbolic reasoning. Among the different types of knowledge representations in the domain of artificial intelligence, logical representations stem from classical logic. However, this is not suitable for representing or treating items of information containing vagueness, incompleteness or uncertainty, or knowledge base evolution that leads the agent to change his beliefs about the world.

When a new item of information is added to a knowledge base, it may become inconsistent. In the argumentation theory people trying to solve the same principle 51314115 in different framework. Revision means modifying the knowledge base in order to maintain consistency [81, while keeping the new information and removing (contraction) or not removing the least possible previous information. In our case, update means revision and contraction, that is insertion and deletion in database perspective. Previous work [6]7 makes connections with contraction from knowledge base dynamics.

\footnotetext{
* This paper extends work from Delhibabu [34] and Mayol [83]
} 
Our knowledge base dynamics is defined in two parts: an immutable part (formulae) and updatable part (literals) (for definition and properties see works of Nebel [88] and Segerberg [98]). Knowledge bases have a set of integrity constraints (see the definitions in later section). In the case of finite knowledge bases, it is sometimes hard to see how the update relations should be modified to accomplish certain knowledge base updates.

Example 1. Consider a database with an (immutable) rule that a staff member is a person who is currently working in a research group under a chair. Additional (updatable) facts are that matthias and gerhard are group chairs, and delhibabu and aravindan are staff members in group info1. Our first integrity constraint (IC) is that each research group has only one chair ie. $\forall x, y, z(\mathrm{y}=\mathrm{x}) \leftarrow$ group_chair $(\mathrm{x}, \mathrm{y}) \wedge$ group_chair(x,z). Second integrity constraint is that a person can be a chair for only one research group ie. $\forall x, y, z(\mathrm{y}=\mathrm{z}) \leftarrow \operatorname{group} \_c h a i r(\mathrm{y}, \mathrm{x})$ $\wedge$ group_chair $(\mathrm{z}, \mathrm{x})$.

Immutable part: staff_chair $(X, Y) \leftarrow$ staff_group $(X, Z)$,group_chair $(Z, Y)$.

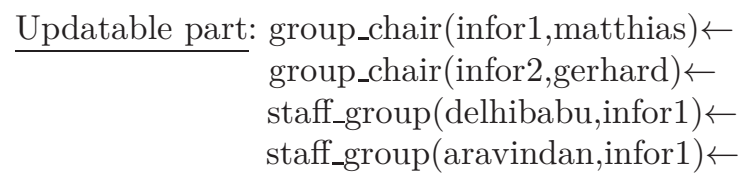

Suppose we want to update this database with the information, staff_chair(aravindan,gerhard); From the immutable part, we can deduce that this can be achieved by asserting staff_group(aravindan, Z) $\wedge$ group_chair(Z,gerhard)

If we are restricted to definite clauses, there are three plausible ways to do this: first case is, aravindan and gerhard belong to infor1, i.e, staff_group (aravindan,-info1) \ group_chair(info1,gerhard). We need to delete both base facts group_chair(infor1,matthias) $\leftarrow$ and group_chair(infor2, gerhard) $\leftarrow$, because our first IC as well as second IC would be violated otherwise. In order to change the view, we need to insert group_chair(infor1,gerhard) $\leftarrow$ as a base fact. Assume that we have an algorithm that deletes the base facts staff_group(delhibabu,infor 1$) \leftarrow$ from the database. But, no rational person will agree with such an algorithm, because the fact staff_group(delhibabu,infor 1$) \leftarrow$ is not "relevant" to the view atom.

Second case, aravindan and gerhard belong to infor2, that is staff_group (aravindan,info2) ^ group_chair(info2,gerhard). Simply, insert the new fact staff_group(aravindan,infor 2$) \leftarrow$ to change the view. Suppose an algorithm deletes the base facts staff_group(aravindan,infor 1$) \leftarrow$ from the database, then it can not be "rational" since these facts are not "relevant" to the view atom.

Third case, aravindan and gerhard belong to infor3 (free assignment of the group value), that is staff_group(aravindan,info3) $\bigwedge$ group_chair(info3,gerhard). Suppose, we insert new base fact group_chair(info3,gerhard) $\leftarrow$, our second IC does not follow. Suppose an algorithm inserts the new base fact staff_group(aravindan,infor 2$) \leftarrow$ or staff_group(aravindan,infor 1$) \leftarrow$ is deleted, then it can not be "rational". 
The above example highlights the need for some kind of "relevance policy" to be adopted when a view atom is to be inserted to a deductive database. How many such axioms and policies do we need to characterize a "good" view update? When are we sure that our algorithm for view update is "rational"? Clearly, there is a need for an axiomatic characterization of view updates. By axiomatic characterization, we mean explicitly listing all the rationality axioms that are to be satisfied by any algorithm for view update.

When dealing with the revision of a knowledge base (both insertions and deletions), there are other ways to change a knowledge base and it has to be performed automatically also. Considering the information, change is precious and must be preserved as much as possible. The principle of minimal change [58 197] can provide a reasonable strategy. On the other hand, practical implementations have to handle contradictory, uncertain, or imprecise information, so several problems can arise: how to define efficient change in the style of Carlos Alchourrón, Peter Gärdenfors, and David Makinson (AGM) [2]; what result has to be chosen 66|72|86; and finally, according to a practical point of view, what computational model to support for knowledge base revision has to be provided?

The basic idea in [118] is to employ the model generation property of hyper tableaux and magic set to generate models, and read off diagnosis from them. One specific feature of this diagnosis algorithm is the use of semantics (by transforming the system description and the observation using an initial model of the correctly working system) in guiding the search for a diagnosis. This semantical guidance by program transformation turns out to be useful for database updates as well. More specifically we use a (least) Herbrand model of the given database to transform it along with the update request into a disjunctive logic program in such a way that the models of this transformed program stand for possible updates. This paper aims at studying the view update algorithms in relational databases. First, we define a framework for highlighting the basic theory of minimal change. Thus, we present a generalized revision algorithm based on abductive explanation for knowledge base revision and main view update method.

The rest of paper is organized as follows: First we start with preliminaries in Section 2. In Section 3, we introduce knowledge base dynamics along with the concept of generalized revision, and revision operator for knowledge base. Section 4 studies the relationship between knowledge base dynamics and abduction. We discuss an important application of knowledge base dynamics in providing an axiomatic characterization for updating view literal to databases. We briefly discuss hyper tableaux calculus and magic set in Section 5. We present two variants of our rational and efficient algorithm for view update in Section 6. In Section 7 , we discuses six basic dimensions in the process of view updating and comparative study of view update algorithms in rational approach is presented. In Section 7, we give brief overview. In Section 8 we draw conclusions with a summary of our contribution and indicate future directions of our investigation. All proofs can be found in the Appendix. 


\section{Preliminaries}

We consider a propositional language $\mathcal{L}_{\mathcal{P}}$ defined from a finite set of propositional variables $\mathcal{P}$ and the standard connectives. We use lower case Roman letters $a, b, x, y, \ldots$ to range over elementary letters and Greek letters $\varphi, \phi, \psi, \ldots$ for propositional formulae. Sets of formulae are denoted by upper case Roman letters $A, B, F, K, \ldots$. A literal is an atom (positive literal), or a negation of an atom (negative literal).

For any formula $\varphi$, we write $E(\varphi)$ to mean the set of the elementary letters that occur in $\varphi$. The same notation also applies to a set of formulae. For any set $F$ of formulae, $L(F)$ represents the sub-language generated by $E(F)$, i.e., the set of all formulae $\varphi$ with $E(\varphi) \subseteq E(F)$.

Horn formulae are defined [37] as follows:

1. Every $a \in \Phi$ where $\Phi \in \mathcal{L}_{\mathcal{P}} \cup\{\perp\}, a$ and $\neg a$ are Horn clauses.

2. $a \leftarrow a_{1} \wedge a_{2} \wedge \ldots \wedge a_{n}$ is a Horn clause, where $n \geq 0$ and $a, a_{i} \in \Phi(1 \leq i \leq n)$.

3. Every Horn clause is a Horn formula, $a$ is called head and $a_{i}$ is body of the Horn formula.

4. If $\varphi$ and $\psi$ are Horn formulae, so is $\varphi \wedge \psi$.

A definite Horn clause is a finite set of literals (atoms) that contains exactly one positive literal which is called the head of the clause. The set of negative literals of this definite Horn clause is called the body of the clause. A Horn clause is non-recursive, if the head literal does not occur in its body. We usually denote a Horn clause as head $\leftarrow$ body. Let $\mathcal{L}_{\mathcal{H}}$ be the set of all Horn formulae with respect to $\mathcal{L}_{\mathcal{P}}$. A formula $\phi$ is a syntactic consequence within $\mathcal{L}_{\mathcal{P}}$ of a set $\Gamma$ of formulas if there is a formal proof in $\mathcal{L}_{\mathcal{P}}$ of $\phi$ from the set $\Gamma$ is $\Gamma \vdash_{\mathcal{L}_{\mathcal{P}}} \phi$.

A immutable part is a function-free clause of the form $a \leftarrow a_{1} \wedge a_{2} \wedge \ldots \wedge a_{n}$, with $n \geq 1$ where $a$ is an atom denoting the immutable part's head and $a_{1} \wedge$ $a_{2} \wedge \ldots \wedge a_{n}$ are literals. i.e., positive or negative atoms, representing the body of the Horn clauses.

Formally, a finite Horn knowledge base $K B$ is defined as a finite set of formulae from language $\mathcal{L}_{\mathcal{H}}$, and divided into three parts: an immutable theory $K B_{I}$ is a Horn formula (head $\leftarrow$ body), which is the fixed part of the knowledge; updatable theory $K B_{U}$ is a Horn clause (head $\leftarrow$ ); and integrity constraint $K B_{I C}$ representing a set of clauses ( $\leftarrow$ body).

Definition 1 (Horn Knowledge Base). A Horn knowledge base, $K B$ is a finite set of Horn formulae from language $\mathcal{L}_{\mathcal{H}}$, s.t $K B=K B_{I} \cup K B_{U} \cup K B_{I C}$, $K B_{I} \cap K B_{U}=\varnothing$ and $K B_{U} \cap K B_{I C}=\varnothing$.

In the AGM framework, a belief set is represented by a deductively closed set of propositional formulae. While such sets are infinite, they can always be finitely representable. However, working with deductively closed, infinite belief sets is not very attractive from a computational point of view. The AGM approach to belief dynamics is very attractive in its capturing the rationality of change, but it is not always easy to implement either Horn formula based partial meet revision. In 
real application from artificial intelligence and databases, what is required is to represent the knowledge using a finite Horn knowledge base. Further, a certain part of the knowledge is treated as immutable and should not be changed.

Knowledge base change deals with situations in which an agent has to modify its beliefs about the world, usually due to new or previously unknown incoming information, also represented as formulae of the language. Common operations of interest in Horn knowledge base change are the expansion of an agent's current Horn knowledge base KB by a given Horn clause $\varphi$ (usually denoted as $\mathrm{KB}+\varphi$ ), where the basic idea is to add regardless of the consequences, and the revision of its current beliefs by $\varphi$ (denoted as $\mathrm{KB} * \varphi$ ), where the intuition is to incorporate $\varphi$ into the current beliefs in some way while ensuring consistency of the resulting theory at the same time. Perhaps the most basic operation in Horn knowledge base change, like belief change, is that of contraction (AGM [2]), which is intended to represent situations in which an agent has to give up $\varphi$ from its current stock of beliefs (denoted as $\operatorname{KB}-\varphi$ ).

Definition 2 (AGM Contraction). Let $K B$ be a Horn knowledge base, and $\alpha$ a belief that is present in $K B$. Then contraction of $K B$ by $\alpha$, denoted as $K B-\alpha$, is a consistent belief set that excludes $\alpha$

Definition 3 (Levi Identity). Let - be an AGM contraction operator for KB. A way to define a revision is by using Generalized Levi Identity:

$$
K B * \alpha=(K B-\neg \alpha) \cup \alpha
$$

Then, the revision can be trivially achieved by expansion, and the axiomatic characterization could be straightforwardly obtained from the corresponding characterizations of the traditional models [45. The aim of our work is not to define revision from contraction, but rather to construct and axiomatically characterize revision operators in a direct way.

\section{Knowledge base dynamics}

AGM 2] proposed a formal framework in which revision(contraction) is interpreted as belief change. Focusing on the logical structure of beliefs, they formulate eight postulates which a revision knowledge base (contraction knowledge base was discussed in [7]) has to verify.

In the AGM approach, a belief is represented by a sentence over a suitable language $\mathcal{L}_{\mathcal{H}}$, and a belief $K B$ is represented by a set of sentence that are close wrt the logical closure operator $\mathrm{Cn}$. It is assumed that $\mathcal{L}_{\mathcal{H}}$, is closed under application of the boolean operators negation, conjunction, disjunction, and implication.

Definition 4. Let $K B$ be a knowledge base with an immutable part $K B_{I}$. Let $\alpha$ and $\beta$ be any two clauses from $\mathcal{L}_{\mathcal{H}}$. Then, $\alpha$ and $\beta$ are said to be KB-equivalent iff the following condition is satisfied: $\forall$ set of Horn clauses $E \subseteq \mathcal{L}_{\mathcal{H}}: K B_{I} \cup E \vdash \alpha$ iff $K B_{I} \cup E \vdash \beta$. 
These postulates stem from three main principles: the new item of information has to appear in the revised knowledge base, the revised base has to be consistent and revision operation has to change the least possible beliefs. Now we consider the revision of a Horn clause $\alpha$ wrt KB, written as $K B * \alpha$. The rationality postulates for revising $\alpha$ from $\mathrm{KB}$ can be formulated as follows:

\section{Definition 5 (Rationality postulates for knowledge base revision).}

$\left(K B^{*} 1\right)$ Closure: $K B * \alpha$ is a knowledge base.

$\left(K B^{*}\right.$ 2) Weak Success: if $\alpha$ is consistent with $K B_{I} \cup K B_{I C}$ then $\alpha \subseteq K B * \alpha$. (KB*3.1) Inclusion: $K B * \alpha \subseteq C n(K B \cup \alpha)$.

(KB*3.2) Immutable-inclusion: $K B_{I} \subseteq C n(K B * \alpha)$.

(KB*4.1) Vacuity 1: if $\alpha$ is inconsistent with $K B_{I} \cup K B_{I C}$ then $K B * \alpha=K B$. (KB*4.2) Vacuity 2: if $K B \cup \alpha \nVdash \perp$ then $K B * \alpha=K B \cup \alpha$.

$\left(K B^{*} 5\right)$ Consistency: if $\alpha$ is consistent with $K B_{I} \cup K B_{I C}$ then $K B * \alpha$ is consistent with $K B_{I} \cup K B_{I C}$.

$\left(K B^{*} 6\right) \quad$ Preservation: If $\alpha$ and $\beta$ are $K B$-equivalent, then $K B * \alpha \leftrightarrow K B * \beta$.

(KB*\%.1) Strong relevance: $K B * \alpha \vdash \alpha$ If $K B_{I} \nvdash \neg \alpha$

(KB*\%.2) Relevance: If $\beta \in K B \backslash K B * \alpha$, then there is a set $K B^{\prime}$ such that $K B * \alpha \subseteq K B^{\prime} \subseteq K B \cup \alpha, K B^{\prime}$ is consistent $K B_{I} \cup K B_{I C}$ with $\alpha$, but $K B^{\prime} \cup\{\beta\}$ is inconsistent $K B_{I} \cup K B_{I C}$ with $\alpha$.

(KB*7.3) Weak relevance: If $\beta \in K B \backslash K B * \alpha$, then there is a set $K B^{\prime}$ such that $K B^{\prime} \subseteq K B \cup \alpha, K B^{\prime}$ is consistent $K B_{I} \cup K B_{I C}$ with $\alpha$, but $K B^{\prime} \cup\{\beta\}$ is inconsistent $K B_{I} \cup K B_{I C}$ with $\alpha$.

To revise $\alpha$ from $\mathrm{KB}$, only those information that are relevant to $\alpha$ in some sense can be added (as the example from the introduction illustrates). ( $K B * 7.1$ ) is a very strong axiom allowing only minimum changes, and certain rational revisions can not be carried out. So, relaxing this condition (example with more details can be found in [7]) allows for weakening strong relevance to relevance only. The relevance policy $(K B * 7.2)$, however, still does not permit rational revisions, so we need to go one step further. With $(K B * 7.3)$ the relevance axiom is further weakened and the resulting conditions are referred to as "coreretainment".

\subsection{Relationship with Abductive Logic Grammars}

The relationship between Horn knowledge base dynamics and abduction was introduced by the philosopher Pierce (see 4). We show how abduction grammar could be used to realize revision with immutability condition. A special subset of literal (atoms) of language $\mathcal{L}_{\mathcal{H}}$, abducibles $\mathrm{Ab}$, are designated for abductive reasoning. Our work is based on atoms, so we combine Christiansen and Dahl 29] grammars method to our theory.

Definition 6 (Abductive grammar). An abductive grammar $\Gamma$ is a 6-tuple $\langle N, T, I C, K B, R, S\rangle$ where

- $N$ are nonterminal symbols in immutable part $\left(K B_{I}\right)$. 
- $T$ is a set of terminal symbols in updatable part $\left(K B_{U}\right)$.

- IC is the integrity constraint to Horn knowledge base $\left(K B_{I C}\right)$.

- $K B$ is the Horn knowledge base which consists of $K B=K B_{I} \cup K B_{U} \cup K B_{I C}$.

- $R$ is a set of rules, $R \subseteq K B$.

- $S$ is the revision of literals (atoms), called the start symbol.

Definition 7 (Constraint system). A constraint system for a abduction is a pair $\left\langle K B^{A b}, K B^{B G}\right\rangle$, where $K B^{A b}(\Delta)$ is a set of propositions (abducibles) and $K B^{B G}$ background Horn knowledge base.

Notations: From grammar point, $K B^{B G}$ is set all Horn formulae from $\mathrm{R}$ and $K B^{A b}$ is set of abducibles from $\mathrm{T}$.

Definition 8 (Minimal abductive explanation). Let KB be a Horn knowledge base and $\alpha$ an observation to be explained. Then, for a set of abducibles $\left(K B^{A b}\right), \Delta$ is said to be an abductive explanation wrt $K B^{B G}$ iff $K B^{B G} \cup \Delta \vdash \alpha$. $\Delta$ is said to be minimal wrt $K B^{B G}$ iff no proper subset of $\Delta$ is an abductive explanation for $\alpha$, i.e. $\nexists \Delta^{\prime}$ s.t. $K B^{B G} \cup \Delta^{\prime} \vdash \alpha$.

Since an incision function is adding and removing only updatable elements from each member of the kernel set, to compute a generalized revision of $\alpha$ from $\mathrm{KB}$, we need to compute only the abduction in every $\alpha$-kernel of KB. So, it is now necessary to characterize precisely the abducibles present in every $\alpha$-kernel of KB. The notion of minimal abductive explanation is not enough to capture this, and we introduce locally minimal and KB-closed abductive explanations.

Definition 9 (Local minimal abductive explanations). Let $K B^{B G^{\prime}}$ be a smallest subset of $K B^{B G}$, s.t $\Delta$ is a minimal abductive explanation of $\alpha$ wrt $K B^{B G^{\prime}}$ (for some $\Delta$ ). Then $\Delta$ is called local minimal for $\alpha$ wrt $K B^{B G}$.

Definition 10 (Constraint abduction system). A constrained abductive grammar is a pair $\langle\Gamma, C\rangle$, where $\Gamma$ is an abductive grammar and $C$ a constraint system for abduction, $\Gamma=\langle N, T, R, S\rangle$ and $C=\left\langle K B^{B G}, K B^{A b}, I C\right\rangle$.

Given a constrained abductive grammar $\langle\Gamma, C\rangle$ as above, the constrained abductive recognition problem for $\tau \in T^{*}$ is the problem of finding an admissible and denial knowledge base from $K B^{A b}$ and such that $\tau \in \mathcal{L}_{P}\left(\Gamma_{K B^{A b}}\right)$ where $\mathcal{L}_{P}\left(\Gamma_{K B^{A b}}\right)$ is propositional language over abducibles in $\Gamma$, where $\Gamma_{K B^{A b}}$ $=\left\langle N, T, K B^{B G} \cup K B^{A b}, R, S\right\rangle$. In this case, $K B^{A b}$ is called a constrained ( $a b$ ductive) system of $\tau$. Such that $K B^{A b}$ is minimal whenever no proper subset of it is an in $\tau$ given $\langle\Gamma, C\rangle$.

Let $K B^{A b} \in\left(\left\{\Delta^{+}, \Delta^{-}\right\}\right)$. Here $\Delta^{+}$refers to admission Horn knowledge base (positive atoms) and $\Delta^{-}$refers to denial Horn knowledge base(negative atoms) wrt given $\alpha$. Then problem of abduction is to explain $\Delta$ with abducibles $\left(K B^{A b}\right)$, s.t. $K B^{B G} \cup \Delta^{+} \cup \Delta^{-} \vdash \alpha$ and $K B^{B G} \cup \Delta^{+} \models \alpha \cup \Delta^{-}$are both consistent with IC. 
Theorem 1. Consider a constrained abductive grammar $A G=\langle\Gamma, C\rangle$ with $\Gamma=\langle N, T, K B, R, S\rangle$ and $C=\left\langle K B^{B G}, K B^{A b}, I C\right\rangle$. Construct a abductive grammar $\Delta(A G)=\left\langle N, T, K B^{B G}, R, S\right\rangle$ by having, for any $\left(\Delta^{+}\right)\left(\right.$or $\left(\Delta^{-}\right)$from $K B^{A b}$, the set of acceptable results for accommodate $\left(\alpha, K B^{B G} \in \Delta^{+}\right)$being of the form $\left(K B^{A b} \backslash \Delta^{+}\right)$where $\left(\Delta^{+} \in K B^{A b^{\prime}}\right) . \Delta^{+}$is a locally minimal set of atoms (literals) $K B^{B G} \cup \Delta^{+}$and $K B^{B G} \cup \Delta^{+}=\alpha$ is consistent with $I C$; if no such $\left(\Delta^{-}\right)$exists (like denial $\left(\Delta^{-}\right)$being of the form $\left(K B^{A b} \backslash \Delta^{-}\right) . \Delta^{-}$is a locally minimal set of atoms (literals) $K B^{B G} \cup \Delta^{-}$and $K B^{B G} \cup \Delta^{-} \models \alpha$ is consistent with $I C)$, otherwise accommodate $\left(\alpha, K B^{B G} \in \Delta^{-}\right)$is not possible.

Now, we need to connect the grammar system $\Gamma$ to the Horn knowledge base $K B$, such that $K B_{I} \cup K B_{U} \cup K B_{I C}=K B^{B G} \cup K B^{A b} \cup I C$ holds. The connection between locally minimal abductive explanation for $\alpha$ wrt $K B_{I}$ and $\alpha$-kernel of KB, which is shown by the following lemma immediately follows from their respective definitions.

\section{Lemma 1.}

1. Let $K B$ be a Horn knowledge base and $\alpha$ a Horn clause s.t. $\nvdash \neg \alpha$. Let $\Delta^{+}$and $\Delta^{-}$be a KB-closed locally minimal abductive explanation for $\alpha$ wrt $K B_{I}$. Then, there exists a $\alpha$-kernel $X$ of $K B$ s.t. $X \cap K B_{U}=\Delta^{+} \cup \Delta^{-}$.

2. Let $K B$ be a Horn knowledge base and $\alpha$ a Horn clause s.t. $\nvdash \neg \alpha$. Let $X$ be a $\alpha$-kernel of $K B$ and $\Delta^{+} \cup \Delta^{-}=X \cap K B_{U}$. Then, $\Delta^{+}$and $\Delta^{-}$are $K B$ -locally minimal abductive explanations for $\alpha$ wrt $K B_{I}$.

An immediate consequence of the above lemma 4.1 is that it is enough to compute all the KB-locally minimal abductive explanations for $\alpha$ wrt $K B_{I}$ in order to revise $\alpha$ from KB. Thus, a well-known abductive procedure to compute an abductive explanation for $\alpha$ wrt $K B_{I}$ could be used.

\subsection{Generalized revision algorithm}

The problem of knowledge base revision is concerned with determining how a request to change can be appropriately translated into one or more atoms or literals. In this section we develop a new generalized revision algorithm. Note that it is enough to compute all the KB-locally minimal abduction explanations for $\alpha$ wrt $K B_{I} \cup K B_{U} \cup K B_{I C}$. If $\alpha$ is consistent with $\mathrm{KB}$ then a well-known abductive procedure for compute an abductive explanation for $\alpha$ wrt $K B_{I}$ could be used to compute kernel revision.

Theorem 2. Let $K B$ be a Horn knowledge base and $\alpha$ is formula.

1. If Algorithm 1 produced $K B^{\prime}$ as a result of revising $\alpha$ from $K B$, then $K B$ ' satisfies all the rationality postulates $\left(K B^{*} 1\right)$ to $\left(K B^{*} 6\right)$ and $\left(K B^{*}\right.$ \%.3).

2. Suppose $K B^{\prime \prime}$ satisfies all these rationality postulates for revising $\alpha$ from $K B$, then $K B^{\prime \prime}$ can be produced by Algorithm 1. 


\begin{tabular}{|c|c|}
\hline Algorithm 1 & Generalized revision algorithm \\
\hline Input : & $\begin{array}{c}\text { A Horn knowledge base } K B=K B_{I} \cup K B_{U} \cup K B_{I C} \\
\text { and a Horn clause } \alpha \text { to be revised. }\end{array}$ \\
\hline Output: & $\begin{array}{c}\text { A new Horn knowledge base } K B^{\prime}=K B_{I} \cup K B_{U}^{*} \cup K B_{I C} \text {, } \\
\text { s.t. } K B^{\prime} \text { is a generalized revision } \alpha \text { to } \mathrm{KB} .\end{array}$ \\
\hline \multicolumn{2}{|c|}{$\begin{array}{l}\text { Procedure } K B(K B, \alpha) \\
\quad \text { begin }\end{array}$} \\
\hline 1. & $\begin{array}{c}\text { Let } \mathrm{V}:=\left\{c \in K B_{I C} \mid K B_{I} \cup K B_{I C} \text { inconsistent with } \alpha \text { wrt } c\right\} \\
\qquad::=N:=\emptyset \text { and } K B^{\prime}=K B\end{array}$ \\
\hline 2. & $\begin{array}{c}\text { While }(V \neq \emptyset) \\
\text { select a subset } V^{\prime} \subseteq V\end{array}$ \\
\hline & $\begin{array}{c}\text { For each } v \in V^{\prime} \text {, select a literal to be } \\
\text { remove (add to } \mathrm{N}) \text { or a literal to be added (add to } \mathrm{P}) \text { wrt } \mathrm{KB} \\
\text { Let } \mathrm{KB}:=K R(K B, P, N) \\
\text { Let } \mathrm{V}:=\left\{c \in K B_{I C} \mid K B_{I} \text { inconsistent with } \alpha \text { wrt } c\right\} \\
\text { return }\end{array}$ \\
\hline $\begin{aligned} & 3 . \\
& \text { end. }\end{aligned}$ & Produce a new Horn knowledge base $K B^{\prime}$ \\
\hline
\end{tabular}

Algorithm 2
Procedure $K R\left(K B, \Delta^{+}, \Delta^{-}\right)$
begin

1. Let $P:=\left\{e \in \Delta^{+}\left|K B_{I}\right| \not e\right\}$ and $N:=\left\{e \in \Delta^{-}\left|K B_{I}\right|=e\right\}$

2. While $(P \neq \emptyset)$ or $(N \neq \emptyset)$

select a subset $P^{\prime} \subseteq P$ or $N^{\prime} \subseteq N$

Construct a set $S_{1}=\{X \mid X$ is a KB-closed locally minimal abductive explanation wrt $\mathrm{P}\}$

Construct a set $S_{2}=\{X \mid X$ is a KB-closed locally minimal abductive explanation wrt N $\}$ Determine hitting set $\sigma\left(S_{1}\right)$ and $\sigma\left(S_{2}\right)$

If $\left(\left(N^{\prime}=\emptyset\right)\right.$ and $\left.\left(P^{\prime} \neq \emptyset\right)\right)$

else

Produce $K B^{\prime}=K B_{I} \cup\left\{\left(K B_{U} \cup \sigma\left(S_{1}\right)\right\}\right.$

end if

Produce $K B^{\prime}=K B_{I} \cup\left\{\left(K B_{U} \backslash \sigma\left(S_{2}\right) \cup \sigma\left(S_{1}\right)\right\}\right.$

If $\left(\left(N^{\prime} \neq \emptyset\right)\right.$ and $\left.\left(P^{\prime}=\emptyset\right)\right)$

Produce $K B^{\prime}=K B_{I} \cup\left\{\left(K B_{U} \backslash \sigma\left(S_{2}\right)\right\}\right.$

else

Produce $K B^{\prime}=K B_{I} \cup\left\{\left(K B_{U} \backslash \sigma\left(S_{2}\right) \cup \sigma\left(S_{1}\right)\right\}\right.$

end if

Let $P:=\left\{e \in \Delta^{+} \mid K B_{I} \not \models e\right\}$ and $N:=\left\{e \in \Delta^{-}\left|K B_{I}\right|=e\right\}$

3. return $K B^{\prime}$

end. 


\section{Deductive database}

A Deductive database $D D B$ consists of three parts: an intensional database IDB $\left(K B_{I}\right)$, a set of definite program clauses, extensional database $E D B\left(K B_{U}\right)$, a set of ground facts; and integrity constraints $I C$. The intuitive meaning of $D D B$ is provided by the Least Herbrand model semantics and all the inferences are carried out through SLD-derivation. All the predicates that are defined in IDB are referred to as view predicates and those defined in $E D B$ are referred to as base predicates. Extending this notion, an atom with a view predicate is said to be a view atom, and similarly an atom with base predicate is a base atom. Further we assume that $I D B$ does not contain any unit clauses and no predicate defined in a given $D D B$ is both view and base.

Two kinds of view updates can be carried out on a $D D B$ : An atom, that does not currently follow from $D D B$, can be inserted, or an atom, that currently follows from $D D B$ can be deleted. When an atom $A$ is to be updated, the view update problem is to insert or delete only some relevant $E D B$ facts, so that the modified $E D B$ together with $I D B$ will satisfy the updating of $A$ to $D D B$.

Note that a $D D B$ can be considered as a knowledge base to be revised. The $I D B$ is the immutable part of the knowledge base, while the $E D B$ forms the updatable part. In general, it is assumed that the language underlying a $D D B$ is fixed and the semantics of $D D B$ is the least Herbrand model over this fixed language. We assume that there are no function symbols implying that the Herbrand Base is finite. Therefore, the $I D B$ is practically a shorthand of its ground instantiation 11 written as $I D B_{G}$. In the sequel, technically we mean $I D B_{G}$ when we refer simply to $I D B$. Thus, a $D D B$ represents a knowledge base where the immutable part is given by $I D B_{G}$ and updatable part is the $E D B$. Hence, the rationality postulates $\left(\mathrm{KB}^{*} 1\right)-\left(\mathrm{KB}^{*} 6\right)$ and $\left(\mathrm{KB}^{*} 7.3\right)$ provide an axiomatic characterization for update (insert and delete) a view atom $A$ from a definite database $D D B$.

Logic provides a conceptual level for understanding the meaning of relational databases. Hence, the rationality postulates $\left(\mathrm{KB}^{*} 1\right)-\left(\mathrm{KB}^{*} 6\right)$ and $\left(\mathrm{KB}^{*} 7.3\right)$ can provide an axiomatic characterization for view updates in relational databases too. A relational database together with its view definitions can be represented by a deductive database $(E D B$ representing tuples in the database and $I D B$ representing the view definitions), and so the same algorithm can be used to delete view extensions from relational deductive databases.

An update request $\mathrm{U}=\mathrm{B}$, where $\mathrm{B}$ is a set of base facts, is not true in $\mathrm{KB}$. Then, we need to find a transaction $T=T_{\text {ins }} \cup T_{\text {del }}$, where $T_{\text {ins }}\left(\Delta_{i}\right)$ (resp. $\left.T_{d e l}\left(\Delta_{j}\right)\right)$ is the set of facts, such that $\mathrm{U}$ is true in $D D B^{\prime}=\left(\left(E D B-T_{\text {del }} \cup\right.\right.$ $\left.\left.T_{\text {ins }}\right) \cup I D B \cup I C\right)$. Since we consider stratifiable (definite) deductive databases, SLD-tree can be used to compute the required abductive explanations. The idea is to get all EDB facts used in a SLD-derivation of $A$ wrt DDB, and construct that as an abductive explanation for $A$ wrt $I D B_{G}$.

\footnotetext{
${ }^{1}$ a ground instantiation of a definite program $P$ is the set of clauses obtained by substituting terms in the Herbrand Universe for variables in $P$ in all possible ways
} 
All solutions translate [85] a view update request into a transaction combining insertions and deletions of base relations for satisfying the request. Further, a stratifiable (definite) deductive database can be considered as a knowledge base, and thus rationality postulates and insertion algorithm of the previous section can be applied for view updates in database.

\section{View update method}

View updating [1] aims at determining one or more base relation updates such that all given update requests with respect to derived relations are satisfied after the base updates have been successfully applied.

Definition 11 (View update). Let $D D B=\langle I D B, E D B, I C\rangle$ be a stratifiable (definite) deductive database $D D B(D)$. A VU request $\nu_{D}$ is a pair $\left\langle\nu_{D}^{+}, \nu_{D}^{-}\right\rangle$where $\nu_{D}^{+}$and $\nu_{D}^{-}$are sets of ground atoms representing the facts to be inserted into $D$ or deleted from $D$, resp., such that pred $\left(\nu_{D}^{+} \cup \nu_{D}^{-}\right) \subseteq \operatorname{pred}(I D B), \nu_{D}^{+} \cap \nu_{D}^{-}=\emptyset$, $\nu_{D}^{+} \cap P M_{D}=\emptyset$ and $\nu_{D}^{-} \subseteq P M_{D}$.

Note that we consider again true view updates only, i.e., ground atoms which are presently not derivable for atoms to be inserted, or are derivable for atoms to be deleted, respectively. A method for view updating determines sets of alternative updates satisfying a given request. A set of updates leaving the given database consistent after its execution is called $V U$ realization.

Definition 12 (Induced update). Let $D D B=\langle I D B, E D B, I C\rangle$ be a stratifiable (definite) deductive database and $D D B=\nu_{D}$ a VU request. A VU realization is a base update $u_{D}$ which leads to an induced update $u_{D \rightarrow D^{\prime}}$ from $D$ to $D^{\prime}$ such that $\nu_{D}^{+} \subseteq P M_{D^{\prime}}$ and $\nu_{D}^{-} \cap P M_{D^{\prime}}=\emptyset$.

There may be infinitely many realizations and even realizations of infinite size which satisfy a given VU request. A breadth-first search (BFS) is employed for determining a set of minimal realizations $\tau_{D}=\left\{u_{D}^{1}, \ldots, u_{D}^{i}\right\}$. Any $u_{D}^{i}$ is minimal in the sense that none of its updates can be removed without losing the property of being a realization for $\nu_{D}$.

In 988 a variant of clausal normal form tableaux called "hyper tableaux" is introduced. Since the hyper tableaux calculus constitutes the basis for our view update algorithm, Clauses, i.e., multisets of literals, are usually written as the disjunction $A_{1} \vee A_{2} \vee \cdots \vee A_{m} \vee \operatorname{not} B_{1} \vee \operatorname{not} B_{2} \cdots \vee \operatorname{not} B_{n}(M \geq 0, n \geq 0)$. The literals $A_{1}, A_{2}, \ldots A_{m}$ (resp. $B_{1}, B_{2}, \ldots, B_{n}$ ) are called the head (resp. body) of a clause. With $\bar{L}$ we denote the complement of a literal $L$. Two literals $L$ and $K$ are complementary if $\bar{L}=K$

From now on $D$ always denotes a finite ground clause set, also called database, and $\Sigma$ denotes its signature, i.e., the set of all predicate symbols occurring in it. We consider finite ordered trees $T$ where the nodes, except the root node, are labeled with literals. In the following we will represent a branch $b$ in $T$ by the sequence $b=L_{1}, L_{2}, \ldots, L_{n}(n \geq 0)$ of its literal labels, where $L_{1}$ labels an 
immediate successor of the root node, and $L_{n}$ labels the leaf of $b$. The branch $b$ is called regular iff $L_{i} \neq L_{j}$ for $1 \leq i, j \leq n$ and $i \neq j$, otherwise it is called irregular. The tree $T$ is regular iff every of its branches is regular, otherwise it is irregular. The set of branch literals of $b$ is $\operatorname{lit}(b)=\left\{L_{1}, L_{2}, \ldots, L_{n}\right\}$. For brevity, we will write expressions like $A \in b$ instead of $A \in l i t(b)$. In order to memorize the fact that a branch contains a contradiction, we allow to label a branch as either open or closed. A tableau is closed if each of its branches is closed, otherwise it is open.

Definition 13 (Hyper Tableau). A literal set is called inconsistent iff it contains a pair of complementary literals, otherwise it is called consistent. Hyper tableaux for $D$ are inductively defined as follows:

Initialization step: The empty tree, consisting of the root node only, is a hyper tableau for $D$. Its single branch is marked as "open".

Hyper extension step: If (1) $T$ is an open hyper tableau for $D$ with open branch $b$, and (2) $C=A_{1} \vee A_{2} \vee \cdots \vee A_{m} \leftarrow B_{1} \wedge B_{2} \cdots \wedge B_{n}$ is a clause from $D(n \geq$ $0, m \geq 0$ ), called extending clause in this context, and (3) $\left\{B_{1}, B_{2}, \ldots, B_{n}\right\} \subseteq b$ (equivalently, we say that $C$ is applicable to $b$ )then the tree $T$ is a hyper tableau for $D$, where $T$ is obtained from $T$ by extension of $b$ by $C$ : replace $b$ in $T$ by the new branches

$$
\left(b, A_{1}\right),\left(b, A_{2}\right), \ldots,\left(b, A_{m}\right),\left(b, \neg B_{1}\right),\left(b, \neg B_{2}\right), \ldots,\left(b, \neg B_{n}\right)
$$

and then mark every inconsistent new branch as "closed", and the other new branches as "open".

The applicability condition of an extension expresses that all body literals have to be satisfied by the branch to be extended. From now on, we consider only regular hyper tableaux. This restriction guarantees that for finite clause sets no branch can be extended infinitely often. Hence, in particular, no open finished branch can be extended any further. This fact will be made use of below occasionally. Notice as an immediate consequence of the above definition that open branches never contain negative literals.

\subsection{View update algorithm}

The key idea of the algorithm presented in this paper is to transform the given database along with the view update request into a disjunctive logic program and apply known disjunctive techniques to solve the original view update problem. The intuition behind the transformation is to obtain a disjunctive logic program in such a way that each (minimal) model of this transformed program represent a way to update the given view atom. We present two variants of our algorithm. The one that is discussed in this section employs a trivial transformation procedure but has to look for minimal models; and another performs a costly transformation, but dispenses with the requirement of computing the minimal models. 


\subsection{Minimality test}

We start presenting an algorithm for stratifiable (definite) deductive databases by first defining precisely how the given database is transformed into a disjunctive logic program for the view deletion process [8] (successful branch - see in Algorithms 3 and 4 via Hyper Tableau).

Definition 14 (IDB Transformation). Given an IDB and a set of ground atoms $S$, the transformation of IDB wrt $S$ is obtained by translating each clause $C \in I D B$ as follows: Every atom $A$ in the body (resp. head) of $C$ that is also in $S$ is moved to the head (resp. body) as $\neg A$.

Note 1. If $I D B$ is a stratifiable deductive database then the transformation introduced above is not necessary.

Definition 15 (IDB* Transformation). Let IDB $\cup E D B$ be a given database. Let $S_{0}=E D B \cup\{A \mid A$ is a ground IDB atom $\}$. Then, IDB* is defined as the transformation of IDB wrt $S_{0}$.

Note 2. Note that $I D B^{*}$ is in general a disjunctive logic program. The negative literals $(\neg A)$ appearing in the clauses are intuitively interpreted as deletion of the corresponding atom $(A)$ from the database. Technically, a literal $\neg A$ is to be read as a positive atom, by taking the $\neg$-sign as part of the predicate symbol. To be more precise, we treat $\neg A$ as an atom wrt $I D B^{*}$, but as a negative literal wrt $I D B$.

Note that there are no facts in $I D B^{*}$. So when we add a delete request such as $\neg A$ to this, the added request is the only fact and any bottom-up reasoning strategy is fully focused on the goal (here the delete request)

Definition 16 (Update Tableaux Hitting Set). An update tableau for a database IDB $\cup E D B$ and delete request $\neg A$ is a hyper tableau $T$ for IDB* $\cup$ $\{\neg A \leftarrow\}$ such that every open branch is finished. For every open finished branch $b$ in $T$ we define the hitting set (of $b$ in $T$ ) as $H S(b)=\{A \in E D B \mid \neg A \in b\}$.

Definition 17 (Minimality test). Let $T$ be an update tableau for IDB $\cup E D B$ and delete request $\neg A$. We say that open finished branch $b$ in $T$ satisfies the strong minimality test iff $\forall s \in H S(b): I D B \cup E D B \backslash H S(b) \cup\{s\} \vdash A$.

Definition 18 (Update Tableau satisfying strong minimality). An update tableau for given $I D B \cup E D B$ and delete request $\neg A$ is transformed into an update tableau satisfying strong minimality by marking every open finished branch as closed which does not satisfy strong minimality.

Next step is view insertion process [11] (For unsuccessful branches - see in Algorithms 3 and 4 via magic set).

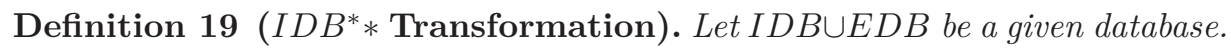
Let $S_{1}=E D B \cup\{A \mid A$ is a ground IDB atom $\}$. Then, IDB** is defined as the transformation of IDB wrt $S_{1}$. 
Note 3. Note that $I D B$ is in general a (stratifiable) disjunctive logic program. The positive literals $(A)$ appearing in the clauses are intuitively interpreted as an insertion of the corresponding atom $(A)$ from the database.

Definition 20 (Update magic Hitting Set). An update magic set rule for a database $I D B \cup E D B$ and insertion request $A$ is a magic set rule $M$ for $I D B^{*} \cup\{A \leftarrow\}$ such that every close branch is finished. For every close finished branch $b$ in $M$ we define the magic set rule (of $b$ in $M$ ) as $H S(b)=\{A \in$ $E D B \mid A \in b\}$.

Definition 21 (Minimality test). Let $M$ be an update magic set rule for $I D B \cup E D B$ and insert request $A$. We say that close finished branch $b$ in $M$ satisfies the strong minimality test iff $\forall s \in H S(b): I D B \cup E D B \backslash H S(b) \cup\{s\} \vdash$ $\neg A$.

Definition 22 (Update magic set rule satisfying strong minimality). An update magic set rule for given IDB $\cup E D B$ and insert request $A$ is transformed into an update magic set rule satisfying strong minimality by marking every close finished branch as open which does not satisfy strong minimality.

This means that every minimal model (minimal wrt the base atoms) of $I D B^{*} \cup\{\neg A\}$ provides a minimal hitting set for deleting the ground view atom $A$. Similarly, $I D B^{*} \cup\{A\}$ provides a minimal hitting set for inserting the ground view atom $A$. Now we are in a position to formally present our algorithm. Given a database and a view atom to be updated, we first transform the database into a definite disjunctive logic program and use hyper tableaux calculus to generate models of this transformed program for deletion of an atom. Second, magic set rule is used to generate models of this transformed program for insertion of an atom. Models that do not represent rational update are filtered out using the strong minimality test. This is formalized in Algorithm 3.

To show the rationality of this approach, we study how this is related to the previous approach presented in the last section, i.e. generating explanations and computing hitting sets of these explanations. To better understand the relationship it is imperative to study where the explanations are in the hyper tableau approach and magic set rule. We first define the notion of $E D B$-cut and then view update seeds.

Definition 23 (EDB-Cut). Let $T$ be update tableau with open branches $b_{1}, b_{2}, \ldots, b_{n}$. $A$ set $S=\left\{A_{1}, A_{2}, \ldots, A_{n}\right\} \subseteq E D B$ is said to be $E D B$-cut of $T$ iff $\neg A_{i} \in b_{i}$ $\left(A_{i} \in b_{i}\right)$, for $1 \leq i \leq n$.

Definition 24 ( $E D B$ seeds). Let $M$ be an update seeds with close branches $b_{1}, b_{2}, \ldots, b_{n}$. A set $S=\left\{A_{1}, A_{2}, \ldots, A_{n}\right\} \subseteq E D B$ is said to be a $E D B$-seeds of $M$ iff EDB seeds vu_seeds $\left(\nu_{D}\right)$ with respect to $\nu_{D}$ is defined as follows:

$$
\text { vu_seeds }\left(\nu_{D}\right):=\left\{\nabla_{p}^{\pi}\left(c_{1}, \ldots, c_{n}\right) \mid p\left(c_{1}, \ldots, c_{n}\right) \in \nu_{D}^{\pi} \text { and } \pi \in\{+,-\}\right\} \text {. }
$$




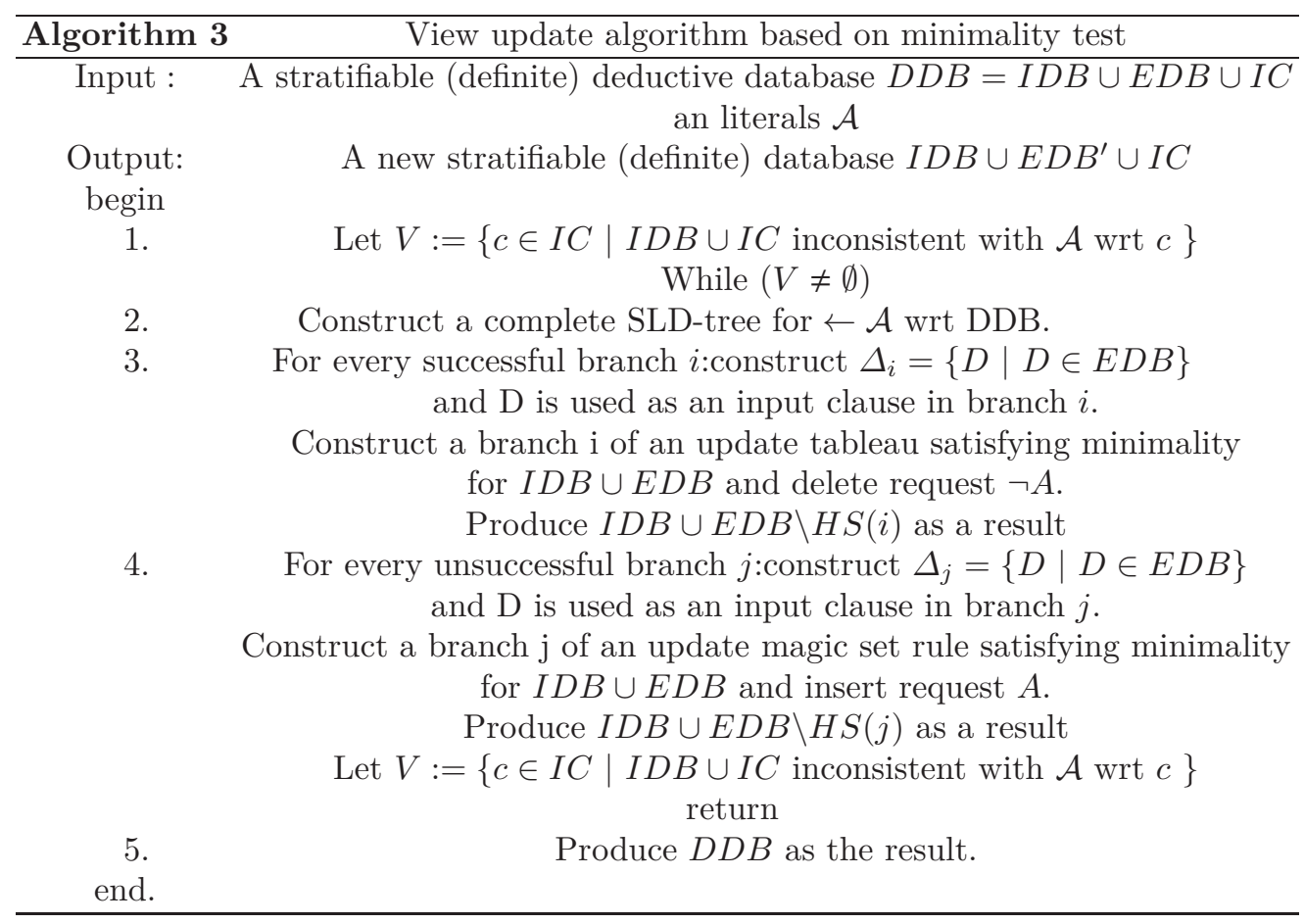

Lemma 2. Let $T$ be an update tableau for $I D B \cup E D B$ and update request $A$. Similarly, for $M$ be an update magic set rule. Let $S$ be the set of all EDBclosed minimal abductive explanations for $A$ wrt. IDB. Let $S^{\prime}$ be the set of all $E D B$-cuts of $T$ and $E D B$-seeds of $M$. Then the following hold

- $S \subseteq S^{\prime}$.

- $\forall \Delta^{\prime} \in S^{\prime}: \exists \Delta \in$ Ss.t. $\Delta \subseteq \Delta^{\prime}$.

The above lemma precisely characterizes what explanations are generated by an update tableau. It is obvious then that a branch cuts through all the explanations and constitutes a hitting set for all the generated explanations. This is formalized below.

Lemma 3. Let $S$ and $S^{\prime}$ be sets of sets s.t. $S \subseteq S^{\prime}$ and every member of $S^{\prime} \backslash S$ contains an element of $S$. Then, a set $H$ is a minimal hitting set for $S$ iff it is a minimal hitting set for $S^{\prime}$.

Lemma 4. Let $T$ be an update tableau for $I D B \cup E D B$ and update request $A$ that satisfies the strong minimality test. Similarly, for $M$ be an update magic set rule. Then, for every open (close) finished branch b in $T, H S(b)(M, H S(b))$ is a minimal hitting set of all the abductive explanations of $A$. 
So, Algorithms 3 generate a minimal hitting set (in polynomial space) of all $E D B$-closed locally minimal abductive explanations of the view atom to be deleted. From the belief dynamics results recalled in section 3, it immediately follows that Algorithms 5 and 6 are rational, and satisfy the strong relevance postulate (KB-7.1).

Theorem 3. Algorithms 3 is a rational, in the sense that they satisfy all the rationality postulates $\left(K B^{*} 1\right)-\left(K B^{*} 6\right)$ and the strong relevance postulate (KB*r.1). Further, any update that satisfies these postulates can be computed by these algorithms.

\subsection{Materialized view}

In many cases, the view to be updates is materialized, i.e., the least Herbrand Model is computed and kept, for efficient query answering. In such a situation, rational hitting sets can be computed without performing any minimality test. The idea is to transform the given $I D B$ wrt the materialized view.

Definition 25 (IDB $B^{+}$Transformation). Let IDB $\cup E D B$ be a given database. Let $S$ be the Least Herbrand Model of this database. Then, $I D B^{+}$is defined as the transformation of IDB wrt $S$.

Note 4. If $I D B$ is a stratifiable deductive database then the transformation introduced above is not necessary.

Definition 26 (Update Tableau based on Materialized view). An update tableau based on materialized view for a database IDB $\cup E D B$ and delete request $\neg A$ is a hyper tableau $T$ for $I D B^{+} \cup\{\neg A \leftarrow\}$ such that every open branch is finished.

Definition 27 (IDB- Transformation). Let IDB $\cup E D B$ be a given database. Let $S_{1}$ be the Least Herbrand Model of this database. Then, IDB- is defined as the transformation of IDB wrt $S_{1}$.

Definition 28 (Update magic set rule based on Materialized view). $A n$ update magic set rule based on materialized view for a database IDB $\cup$ EDB and insert request $A$ is a magic set $M$ for $I D B^{+} \cup\{A \leftarrow\}$ such that every close branch is finished.

Now the claim is that every model of $I D B^{+} \cup\{\neg A \leftarrow\}(A \leftarrow)$ constitutes a rational hitting set for the deletion and insertion of the ground view atom $A$. So, the algorithm works as follows: Given a database and a view update request, we first transform the database wrt its Least Herbrand Model (computation of the Least Herbrand Model can be done as a offline preprocessing step. Note that it serves as materialized view for efficient query answering). Then the hyper tableaux calculus (magic set rule) is used to compute models of this transformed program. Each model represents a rational way of accomplishing the given view update request. This is formalized in Algorithms 4. 
This approach for view update may not satisfy (KB*7.1) in general. But, as shown in the sequel, conformation to $\left(\mathrm{KB}^{*} 6.3\right)$ is guaranteed and thus this approach results in rational update.

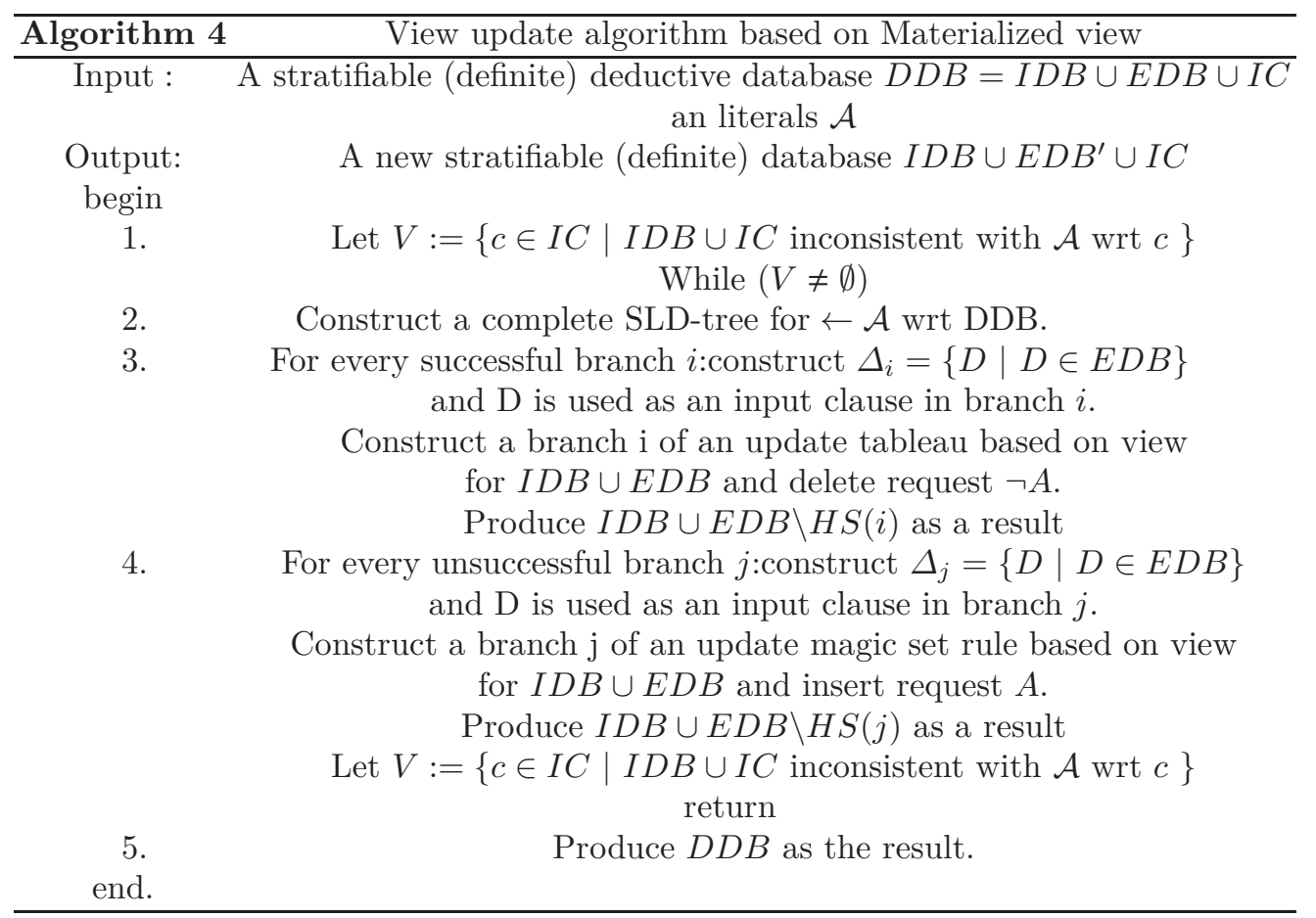

Lemma 5. Let $T$ be an update tableau based on materialized view for IDB $\cup$ $E D B$ and delete request $\neg A(A)$, Similarly, for $M$ be an update magic set rule. Let $S$ be the set of all EDB-closed locally minimal abductive explanations for $A$ wrt IDB. Let $S^{\prime}$ be the set of all EDB-cuts of $T$ and EDB-seeds of $M$. Then, the following hold:

- $S \subseteq S^{\prime}$

- $\forall \bar{\Delta}^{\prime} \in S^{\prime}: \exists \Delta \in S$ s.t. $\Delta \subseteq \Delta^{\prime}$.

- $\forall \Delta^{\prime} \in S^{\prime}: \Delta^{\prime} \subseteq \bigcup S$.

Lemma 6. Let $S$ and $S^{\prime}$ be sets of sets s.t. $S \in S^{\prime}$ and for every member $X$ of $S^{\prime} \backslash S: X$ contains a member of $S$ and $X$ is contained in $\bigcup S$. Then, a set $H$ is a hitting set for $S$ iff it is a hitting set for $S^{\prime}$.

Lemma 7. Let $T$ and $M$ be defined as in Lemma 5. Then $H S(b)$ is a rational hitting set for $A$, for every open finished branch $b$ in $T$ (close finished branch $b$ in $M)$.

Theorem 4. Algorithms 4 is a rational, in the sense that they satisfy all the rationality postulates $\left(K B^{*} 1\right)$ to $\left(K B^{*} 6\right)$ and $\left(K B^{*} \% .3\right)$. 


\section{A Comparative Study of Integrity Constraints and View Update}

During the process of updating a database, two interrelated problems could arise. On one hand, when an update is applied to the database, integrity constraints could become inconsistent with request, then stop the process. On the other hand, when an update request consist on updating some derived predicate, a view updating mechanism must be applied to translate the update request into correct updates on the underlying base facts. Our work is not focusing on the integrity constraint maintenance approach. In this section, we extend Mayol and Teniente's 83 survey for view updating and integrity constraint.

The main aspects that must be taken into account during the process of view updating and integrity constraint [48] enforcement are the following: the problem addressed, the considered database schema, the allowed update requests, the used technique, update change and the obtained solutions. These six aspects provide the basic dimensions to be taken into account. We explain each dimension in this section.

\section{Problem Addressed}

(Type) - What kind of program to be used (stratified(S), Horn clause(H), Disjunctive database(D), Normal Logic program(N) and Other $(\mathrm{O}))$.

(View Update) - Whether they are able to deal with view updating or not (indicated by Yes or No in the second column of Table 1).

(integrity-constraint Enforcement) - Whether they incorporate an integrity constraint checking or an integrity constraint maintenance approach (indicated by check or maintain in the third column).

(Run/Comp) - Whether the method follows a run-time(transaction) or a compiletime approach (indicated by Run or Compile in the fourth column).

\section{Database Schema Considered}

(Definition Language) - The language mostly used is logic, although some methods use a relational language and also uses an object-oriented.

(The DB Schema Contains Views) - All methods that deal with view updating need views to be defined in the database schema. Some of other method allow to define views.

(Restrictions Imposed on the Integrity Constraints) - Some proposals impose certain restrictions on the kind of integrity constraints that can be defined and, thus, handled by their methods.

(Static vs Dynamic Integrity Constraints) - Integrity constraints may be either static, and impose restrictions involving only a certain state of the database, or dynamic.

\section{Update Request Allowed}

(Multiple Update Request) - An update request is multiple if it contains several updates to be applied together to the database. 
(Update Operators) - Traditionally, three different basic update operators are distinguished: insertion $(\iota)$, deletion $(\delta)$ and modification $(\chi)$. Modification can always be simulated by a deletion followed by an insertion.

\section{Update Processing Mechanism}

(Applied Technique) - The techniques applied by these methods can be classified according to four different kinds of procedures, unfolding, SLD, active and predefined programs, respectively.

(Taking Base Facts into Account - Base facts can either be taken into account or not during update processing.

(User Participation) - User participation during update processing or not.

\section{Update Changing Mechanism}

(Type of modification) - Changing table by singleton like atom (S), sets of each types of modification(SS) and group of changes(G).

(Changing Base Fact) - Base fact can be changed either using principle of minimal change or complete change (maximal change).

(Changing View Definition) - Whether update process view definition is changed or not.

\section{Obtained Solution}

(Our Axiom follow) - When update process done, we are comparing our axiomatized method and which relevance policy holds $\left(\left(\mathrm{KB}^{*} 1\right)\right.$ to $\left(\mathrm{KB}^{*} 6\right),\left(\mathrm{KB}^{*} 7.1\right),\left(\mathrm{KB}^{*} 7.2\right)$ and $\left(\mathrm{KB}^{*} 7.3\right)$ is enumerated 1 to 9$)$

(Soundness) - A method is correct if it only obtains solutions that satisfy the requested update.

(Completeness) - A method is complete if it is able to obtain all solutions that satisfy a given update request.

Results of each method according to these features are summarized in Appendix Table 1.

\section{Related Works}

We begin by recalling previous work on view deletion. Chandrabose [617] and Delhibabu 34|35|36, defines a contraction and revision operator in view deletion with respect to a set of formulae or sentences using Hansson's [54] belief change. Similar to our approach, he focused on set of formulae or sentences in knowledge base revision for view update wrt. insertion and deletion and formulae are considered at the same level. Chandrabose proposed different ways to change knowledge base via only database deletion, devising particular postulate which is shown to be necessary and sufficient for such an update process.

Our Horn knowledge base consists of two parts, immutable part and updatable part, but focus is on principle of minimal change. There are more related works on that topic. Eiter [42, Langlois 67, and Delgrande [37] are focusing 
on Horn revision with different perspectives like prime implication, logical closure and belief level. Segerberg 98 defined new modeling for belief revision in terms of irrevocability on prioritized revision. Hansson [54], constructed five types of non-prioritized belief revision. Makinson [77 developed dialogue form of revision AGM. Papini [89] defined a new version of knowledge base revision. Here, we consider immutable part as a Horn clause and updatable part as an atom(literals).

We are bridging gap between philosophical work, paying little attention to computational aspects of database work. In such a case, Hansson's 54 kernel change is related with abductive method. Aliseda's [4 book on abductive reasoning is one of the motivation keys. Christiansen's [29] work on dynamics of abductive logic grammars exactly fits our minimal change (insertion and deletion). Wrobel's [102 definition of first order theory revision was helpful to frame our algorithm.

On other hand, we are dealing with view update problem. Keller's 62 thesis is motivation for view update problem. There is a lot of papers on view update problem (for example, recent survey paper on view update by Chen and Liao 24, survey paper on view algorithm by Mayol and Teniente [83] and current survey paper on view selection (38|53/73|69|79/105). More similar to our work is paper presented by Bessant et al. [16, local search-based heuristic technique that empirically proves to be often viable, even in the context of very large propositional applications. Laurent et al. 68 parented updating deductive databases in which every insertion or deletion of a fact can be performed in a deterministic way.

Furthermore, and at a first sight more related to our work, some work has been done on ontology systems and description logics (Qi and Yang [90, and Kogalovsky [63]). Finally, when we presented connection between belief update versus database update, we did not talk about complexity (see the works of Liberatore [70171], Caroprese [19], Calvanese's [20, and Cong [31]).

The significance of our work can be summarized in the following:

- We have defined new way of insertion and deletion of an atom(literals) as per norm of principle of minimal change.

- We have proposed new generalized revision algorithm for knowledge base dynamics, interesting connections with kernel change and abduction procedure.

- We have written new view update algorithm for DDB, and we provided stratifiable (definite) deductive database, using our axiomatic method based on Hyper tableaux and magic sets.

- Finally, we presented current Comparative Study of view update algorithms.

\section{Conclusion and remarks}

The main contribution of this research is to provide a link between theory of belief dynamics and concrete applications such as view updates in databases. We argued for generalization of belief dynamics theory in two respects: to handle certain part of knowledge as immutable; and dropping the requirement that belief state be deductively closed. The intended generalization was achieved by 
introducing the concept of knowledge base dynamics and generalized contraction for the same. Further, we also studied the relationship between knowledge base dynamics and abduction resulting in a generalized algorithm for revision based on abductive procedures. We also successfully demonstrated how knowledge base dynamics can provide an axiomatic characterization for updating an atom(literals) to a stratifiable (definite) deductive database.

In bridging the gap between belief dynamics and view updates, we have observed that a balance has to be achieved between computational efficiency and rationality. While rationally attractive notions of generalized revision prove to be computationally inefficient, the rationality behind efficient algorithms based on incomplete trees is not clear at all. From the belief dynamics point of view, we may have to sacrifice some postulates, vacuity for example, to gain computational efficiency. Further weakening of relevance has to be explored, to provide declarative semantics for algorithms based on incomplete trees.

On the other hand, from the database side, we should explore various ways of optimizing the algorithms that would comply with the proposed declarative semantics. We believe that partial deduction and loop detection techniques, will play an important role in optimizing algorithms of the previous section. Note that, loop detection could be carried out during partial deduction, and complete SLD-trees can be effectively constructed wrt a partial deduction (with loop check) of a database, rather than wrt database itself. Moreover, we would anyway need a partial deduction for optimization of query evaluation.

We have presented two variants of an algorithm for updating a view atom to a definite database. The key idea of this approach is to transform the given database into a disjunctive logic program in such a way that updates can be read off from the models of this transformed program. One variant based on materialized views is of polynomial time complexity. Moreover, we have also shown that this algorithm is rational in the sense that it satisfies the rationality postulates that are justified from philosophical angle.

In the second variant, where materialized view is used for the transformation, after generating a hitting set and removing corresponding $E D B$ atoms, we easily move to the new materialized view. An obvious way is to recompute the view from scratch using the new $E D B$ (i.e. compute the Least Herbrand Model of the new updated database from scratch) but it is certainly interesting to look for more efficient methods. In the end, we plan to redefined the model to Horn Logic with stratified Negation [60] and Argumentative Inference [6/57.

Though we have discussed only about view updates, we believe that knowledge base dynamics can also be applied to other applications such as view maintenance, diagnosis, and we plan to explore it further (see works [19] and [18]). It would also be interesting to study how results using soft stratification 11 with belief dynamics, especially the relational approach, could be applied in real world problems. Still, a lot of developments are possible, for improving existing operators or for defining new classes of change operators. As immediate extension, question raises: is there any real life application for AGM in 25 year theory? 47. The revision and update are more challenging in logical view update prob- 
lem(database theory), so we can extend the theory to combine results similar to Konieczny's 64] and Nayak's 87.

\section{Appendix}

Proof of Theorem 1. Sound and Completeness are trivially to shown from the definition.

Proof of Lemma 1.

1. The fact that $\nvdash \neg \alpha$ and there exists a KB - closed locally minimal abductive explanation for $\alpha$ wrt $K B_{I}$, it is clear that there exists at least one $\alpha$ kernel of KB. Suppose $\Delta\left(\Delta \in \Delta^{+} \cup \Delta^{-}\right)$is empty (i.e., $\left.K B_{I} \vdash \neg \alpha\right)$, then the required result follows immediately. If not, since $\Delta$ is a locally minimal abductive explanation, there exists a minimal subset $K B_{I}^{\prime} \subseteq K B_{I}$, s.t. $\Delta$ is minimal abductive explanation of $\alpha$ wrt $K B_{I}^{\prime}$. Since, $\Delta$ is KB-closed, it is not difficult to see that $K B_{I}^{\prime} \cup \Delta^{+} \cup \Delta^{-}$is a $\alpha$ - kernel of KB.

2. Since $\mathrm{X}$ is a $\alpha$ - kernel of $\mathrm{KB}$ and $\Delta$ is the set of all abducibles in $\mathrm{X}$, it follows that $\Delta^{+} \cup \Delta^{-}$is a minimal abductive explanation of $\Delta$ wrt $X \backslash \Delta^{-} \cup \Delta^{+}$. It is obvious that $\Delta^{+} \cup \Delta^{-}$is KB- closed, and so $\Delta$ is a KB-closed locally minimal abductive explanation for $\alpha$ wrt $K B_{I}$.

Proof of Theorem 2. Sound and Completeness are trivially to shown from the Algorithm 1.

Proof of Lemma 2 and 5.

1. Consider a $\Delta\left(\Delta \in \Delta_{i} \cup \Delta_{j}\right) \in S$. We need to show that $\Delta$ is generated by algorithm 3 at step 2 . From lemma 1 , it is clear that there exists a $A$-kernel $X$ of $D D B_{G}$ s.t. $X \cap E D B=\Delta_{j}$ and $X \cup E D B=\Delta_{i}$. Since $X \vdash A$, there must exist a successful derivation for $A$ using only the elements of $X$ as input clauses and similarly $X \nvdash A$. Consequently $\Delta$ must have been constructed at step 2 .

2. Consider a $\Delta^{\prime}\left(\left(\Delta^{\prime} \in \Delta_{i} \cup \Delta_{j}\right) \in S^{\prime}\right.$. Let $\Delta^{\prime}$ be constructed from a successful(unsuccessful) branch $i$ via $\Delta_{i}\left(\Delta_{j}\right)$. Let $X$ be the set of all input clauses used in the refutation $i$. Clearly $X \vdash A(X \nvdash A)$. Further, there exists a minimal (wrt set-inclusion) subset $Y$ of $X$ that derives $A$ (i.e. no proper subset of $Y$ derives $A)$. Let $\Delta=Y \cap E D B(Y \cup E D B)$. Since IDB does not(does) have any unit clauses, $Y$ must contain some EDB facts, and so $\Delta$ is not empty (empty) and obviously $\Delta \subseteq \Delta^{\prime}$. But, $Y$ need not (need) be a $A$-kernel for $I D B_{G}$ since $Y$ is not ground in general. But it stands for several $A$-kernels with the same (different) EDB facts $\Delta$ in them. Thus, from lemma $1, \Delta$ is a DDB-closed locally minimal abductive explanation for $A$ wrt $I D B_{G}$ and is contained in $\Delta^{\prime}$.

3. Since this proof easy to see materialized view update with minimal.

Proof of Lemma 3 and 6. 
1. (Only if part) Suppose $H$ is a minimal hitting set for $S$. Since $S \subseteq S^{\prime}$, it follows that $H \subseteq \bigcup S^{\prime}$. Further, $H$ hits every element of $S^{\prime}$, which is evident from the fact that every element of $S^{\prime}$ contains an element of $S$. Hence $H$ is a hitting set for $S^{\prime}$. By the same arguments, it is not difficult to see that $H$ is minimal for $S^{\prime}$ too.

(If part) Given that $H$ is a minimal hitting set for $S^{\prime}$, we have to show that it is a minimal hitting set for $S$ too. Assume that there is an element $E \in H$ that is not in $\bigcup S$. This means that $E$ is selected from some $Y \in S^{\prime} \backslash S$. But $Y$ contains an element of $S$, say $X$. Since $X$ is also a member of $S^{\prime}$, one member of $X$ must appear in $H$. This implies that two elements have been selected from $Y$ and hence $H$ is not minimal. This is a contradiction and hence $H \subseteq \bigcup S$. Since $S \subseteq S^{\prime}$, it is clear that $H$ hits every element in $S$, and so $H$ is a hitting set for $S$. It remains to be shown that $H$ is minimal. Assume the contrary, that a proper subset $H^{\prime}$ of $H$ is a hitting set for $S$. Then from the proof of the only if part, it follows that $H^{\prime}$ is a hitting set for $S^{\prime}$ too, and contradicts the fact that $H$ is a minimal hitting set for $S^{\prime}$. Hence, $H$ must be a minimal hitting set for $S$.

2. (If part) Given that $H$ is a hitting set for $S^{\prime}$, we have to show that it is a hitting set for $S$ too. First of all, observe that $\bigcup S=\bigcup S^{\prime}$, and so $H \subseteq \bigcup S$. Moreover, by definition, for every non-empty member $X$ of $S^{\prime}$, $H \cap X$ is not empty. Since $S \subseteq S^{\prime}$, it follows that $H$ is a hitting set for $S$ too.

(Only if part) Suppose $H$ is a hitting set for $S$. As observed above, $H \subseteq$ $\bigcup S^{\prime}$. By definition, for every non-empty member $X \in S, X \cap H$ is not empty. Since every member of $S^{\prime}$ contains a member of $S$, it is clear that $H$ hits every member of $S^{\prime}$, and hence a hitting set for $S^{\prime}$.

Proof of Lemma 4 and 7. Follows from the lemma 3,4 (minimal test) and 6,7 (materialized view) of [1]

Proof of Theorem 3. Follows from Lemma 3 and Theorem 2.

Proof of Theorem 4. Follows from Lemma 6 and Theorem 2.

\section{Acknowledgement}

The author acknowledges the support of RWTH Aachen, where he is visiting scholar with an Erasmus Mundus External Cooperation Window India4EU by the European Commission when the paper was written. I would like to thanks Chandrabose Aravindan and Gerhard Lakemeyer both my Indian and Germany $\mathrm{PhD}$ supervisor, give encourage to write the paper. 
Appendix B Tab. 1. Summary of view-update and integrity constraint with our axiomatic method

\begin{tabular}{|c|c|c|c|c|c|c|c|c|c|c|c|c|c|c|c|c|c|c|c|}
\hline \multirow[b]{2}{*}{ Method } & \multicolumn{4}{|c|}{ Problem } & \multicolumn{4}{|c|}{ Database schema } & \multicolumn{2}{|c|}{ Update req. } & \multicolumn{3}{|c|}{ Mechanism } & \multicolumn{3}{|c|}{ Update Change } & \multicolumn{3}{|c|}{ Solutions } \\
\hline & Type & \begin{tabular}{|l|} 
View \\
Update
\end{tabular} & $\begin{array}{l}\text { IC } \\
\text { Enforce. }\end{array}$ & $\begin{array}{l}\text { Run/ } \\
\text { Comp. }\end{array}$ & $\begin{array}{l}\text { Def. } \\
\text { Lang. }\end{array}$ & View & \begin{tabular}{|l|} 
IC \\
def.
\end{tabular} & $\begin{array}{l}\text { Kind of } \\
\text { IC }\end{array}$ & Mul. & \begin{tabular}{|l|} 
Update \\
Operat.
\end{tabular} & $\begin{array}{l}\text { Tech- } \\
\text { nique }\end{array}$ & \begin{tabular}{|l|} 
Base \\
Facts \\
\end{tabular} & \begin{tabular}{|l} 
User \\
Part.
\end{tabular} & Type & \begin{tabular}{|c|} 
Base \\
facts
\end{tabular} & \begin{tabular}{|l|} 
View \\
def.
\end{tabular} & Axiom & Sound. & Complete. \\
\hline 50 ] & $\mathrm{N}$ & Yes & Check & Run & Logic & Yes & Yes & Static & No & $\iota \delta$ & SLDNF & No & No & $\mathrm{S}$ & Yes & No & $1-6,9$ & No & \begin{tabular}{|l|}
$\begin{array}{l}\text { Not } \\
\text { proved }\end{array}$ \\
\end{tabular} \\
\hline 61 & $\mathrm{~N}$ & Yes & Maintain & Run & Logic & Yes & Yes & Static & Yes & $\iota \delta$ & SLDNF & No & No & $\mathrm{S}$ & Yes & No & - & No & No \\
\hline 65 & $\mathrm{~S}$ & Yes & Check & Run & Logic & Yes & Yes & Static & Yes & $\iota \delta$ & - & Yes & No & SS & Yes & Yes & $1-6,7$ & \begin{tabular}{|l|} 
Not \\
proved
\end{tabular} & $\begin{array}{l}\text { Not } \\
\text { proved }\end{array}$ \\
\hline 84 & $\mathrm{~N}$ & No & Maintain & Run & Logic & Yes & Yes & Static & Yes & $\iota \delta$ & - & Yes & No & $\mathrm{S}$ & Yes & No & $1-6,7$ & \begin{tabular}{|l|} 
No \\
proved
\end{tabular} & $\begin{array}{l}\text { No } \\
\text { proved }\end{array}$ \\
\hline [51] & S & Yes & $\begin{array}{l}\text { Check } \\
\text { Maintain }\end{array}$ & $\begin{array}{l}\text { Comp. } \\
\text { Run }\end{array}$ & $\begin{array}{l}\text { Relation. } \\
\text { Logic }\end{array}$ & $\mathrm{No}$ & No & Static & Yes & $\iota \delta \chi$ & \begin{tabular}{|l|} 
predef. \\
Programs
\end{tabular} & Yes & No & G & Yes & No & - & No & No \\
\hline 39 & $\mathrm{~N}$ & Yes & Check & Run & Logic & Yes & $\mathrm{No}$ & Static & Yes & $\iota \delta$ & \begin{tabular}{|l|} 
predef \\
Programs
\end{tabular} & Yes & No & S & Yes & No & $1-6,7$ & \begin{tabular}{|l|} 
Not \\
Proved \\
\end{tabular} & No \\
\hline 101 & S & Yes & \begin{tabular}{|l|} 
Check \\
Maintain
\end{tabular} & Run & Logic & Yes & Yes & Static & Yes & $\iota \delta \chi$ & SLDNF & No & No & SS & Yes & No & $1-6,7$ & Yes & No \\
\hline 52 & $\mathrm{~N}$ & Yes & Maintain & Run & Logic & Yes & No & Static & Yes & $\iota \delta$ & Unfold & Yes & No & SS & Yes & No & $1-6,9$ & Yes & Yes \\
\hline 82 & $\mathrm{~N}$ & Yes & Maintain & $\begin{array}{l}\text { Comp. } \\
\text { Run }\end{array}$ & Logic & Yes & Yes & \begin{tabular}{|l|} 
Static \\
Dynamic
\end{tabular} & Yes & $\iota \delta \chi$ & SLDNF & Yes & No & S & Yes & No & $1-6,9$ & \begin{tabular}{|l|} 
Not \\
proved
\end{tabular} & $\begin{array}{l}\text { Not } \\
\text { proved }\end{array}$ \\
\hline 103 & S & Yes & Maintain & Run & Logic & Yes & Yes & Static & Yes & $\iota \delta$ & Unfold. & No & Yes & S & Yes & No & $1-6,7$ & \begin{tabular}{|l|}
$\begin{array}{l}\text { Not } \\
\text { proved }\end{array}$ \\
\end{tabular} & No \\
\hline [6] & $\mathrm{H}$ & Yes & Check & Run & Logic & Yes & Yes & Static & Yes & $\iota \delta$ & SLD & Yes & No & $\mathrm{S}$ & Yes & No & $1-6,9$ & Yes & Yes \\
\hline 22 & $\mathrm{~N}$ & No & Maintain & $\begin{array}{l}\text { Comp } \\
\text { Run }\end{array}$ & $\begin{array}{l}\text { Relation } \\
\text { Logic }\end{array}$ & Yes & Limited & Static & Yes & $\iota \delta \chi$ & Active & Yes & Yes & S & Yes & No & - & No & No \\
\hline 49 & $\mathrm{~N}$ & No & Maintain & \begin{tabular}{|l|} 
Comp \\
Run \\
\end{tabular} & $\begin{array}{l}\text { Relation } \\
\text { Logic } \\
\end{array}$ & No & \begin{tabular}{|l|} 
Flat \\
Limited
\end{tabular} & \begin{tabular}{|l|} 
Static \\
Dynamic \\
\end{tabular} & Yes & $\iota \delta \chi$ & Active & Yes & Yes & $S$ & Yes & No & - & No & No \\
\hline 25 & $\mathrm{H}$ & Yes & $\begin{array}{l}\text { Check } \\
\text { Maintain }\end{array}$ & $\begin{array}{l}\text { Comp. } \\
\text { Run }\end{array}$ & $\mathrm{O}-\mathrm{O}$ & \begin{tabular}{|l|} 
Class \\
Att.
\end{tabular} & Limited & Static & Yes & $\iota \delta$ & Active & Yes & No & SS & Yes & No & $1-6,9$ & No & Yes \\
\hline 32 & $\mathrm{~N}$ & Yes & Maintain & Run & Logic & Yes & \begin{tabular}{|l|} 
Flat \\
Limited \\
\end{tabular} & Static & Yes & $\iota \delta$ & Unfold. & Yes & No & S & Yes & No & $1-6,9$ & \begin{tabular}{|l|} 
Not \\
proved
\end{tabular} & Yes \\
\hline [74] & $\mathrm{N}$ & Yes & Maintain & Run & Logic & Yes & Limited & Static & No & $\iota \delta$ & Active & Yes & No & SS & Yes & No & $1-6,7$ & Yes & $\begin{array}{l}\begin{array}{l}\text { Not } \\
\text { proved }\end{array} \\
\end{array}$ \\
\hline 100 & $\mathrm{~N}$ & Yes & Maintain & $\begin{array}{l}\text { Comp } \\
\text { Run }\end{array}$ & Logic & Yes & Yes & \begin{tabular}{|l|} 
Static \\
Dynamic \\
\end{tabular} & Yes & $\iota \delta$ & SLDNF & Yes & No & S & Yes & No & $1-6,9$ & Yes & Yes \\
\hline 94 & S & Yes & Maintain & Comp & Logic & No & \begin{tabular}{|l|} 
Flat \\
Limited
\end{tabular} & Static & Yes & $\iota \delta$ & \begin{tabular}{|l|} 
predef \\
Programs
\end{tabular} & - & Yes & $G$ & No & Yes & - & No & \begin{tabular}{|l} 
Not \\
proved
\end{tabular} \\
\hline
\end{tabular}




\begin{tabular}{|c|c|c|c|c|c|c|c|c|c|c|c|c|c|c|c|c|c|c|c|}
\hline \multirow[b]{2}{*}{ Method } & \multicolumn{4}{|c|}{ Problem } & \multicolumn{4}{|c|}{ Database schema } & \multicolumn{2}{|c|}{ Update req. } & \multicolumn{3}{|c|}{ Mechanism } & \multicolumn{3}{|c|}{ Update Change } & \multicolumn{3}{|c|}{ Solutions } \\
\hline & Type & \begin{tabular}{|l|} 
View \\
Update \\
\end{tabular} & \begin{tabular}{|l|}
$\mathrm{IC}$ \\
Enforce. \\
\end{tabular} & \begin{tabular}{|l|} 
Run/ \\
Comp.
\end{tabular} & \begin{tabular}{|l|} 
Def. \\
Lang.
\end{tabular} & View & \begin{tabular}{|l|l} 
IC \\
def.
\end{tabular} & $\begin{array}{l}\text { Kind of } \\
\text { IC }\end{array}$ & Mul. & \begin{tabular}{|l|} 
Update \\
Operat. \\
\end{tabular} & \begin{tabular}{|l|} 
Tech- \\
nique
\end{tabular} & \begin{tabular}{|l|} 
Base \\
Facts \\
\end{tabular} & \begin{tabular}{|l|} 
User \\
Part.
\end{tabular} & Type & \begin{tabular}{|c|} 
Base \\
facts
\end{tabular} & \begin{tabular}{|l|} 
View \\
def. \\
\end{tabular} & Axiom & Sound. & Complete. \\
\hline 99 & $\mathrm{~N}$ & No & Maintain & Comp & Logic & Yes & Limited & Static & Yes & $\iota \delta \chi$ & \begin{tabular}{|l|} 
Predef \\
Program \\
\end{tabular} & Yes & No & S & Yes & No & $1-6,7$ & Yes & No \\
\hline 8 & $\mathrm{H}$ & Yes & Check & Run & Logic & Yes & Limited & Static & Yes & $\iota \delta$ & SLD & Yes & No & S & Yes & No & $1-6,9$ & Yes & Yes \\
\hline 33 & $\mathrm{~N}$ & Yes & Maintain & Run & Logic & Yes & Yes & Static & Yes & $\iota \delta$ & SLDNF & No & No & S & Yes & No & $1-6,7$ & $\mathrm{No}$ & $\begin{array}{l}\text { Not } \\
\text { Proved }\end{array}$ \\
\hline 72 & $\mathrm{~N}$ & Yes & Maintain & Run & Logic & Yes & \begin{tabular}{|l|} 
Flat \\
Limited \\
\end{tabular} & Static & Yes & $\iota \delta$ & Unfold & No & Yes & G & Yes & No & $1-6,7$ & \begin{tabular}{|l|}
$\begin{array}{l}\text { Not } \\
\text { proved }\end{array}$ \\
\end{tabular} & No \\
\hline 104. & $\mathrm{H}$ & No & Maintain & \begin{tabular}{|l|} 
Comp. \\
Run
\end{tabular} & Relation & Yes & Limited & \begin{tabular}{|l|} 
Static \\
Dynamic
\end{tabular} & Yes & $\iota \delta \chi$ & Unfold & Yes & No & S & Yes & No & $1-6,7$ & \begin{tabular}{|l|} 
Not \\
proved
\end{tabular} & $\begin{array}{l}\text { Not } \\
\text { proved }\end{array}$ \\
\hline [76 & $\mathrm{N}$ & No & \begin{tabular}{|l|} 
Maintain \\
Restore \\
\end{tabular} & \begin{tabular}{|l|} 
Comp \\
Run
\end{tabular} & Logic & No & \begin{tabular}{|l|} 
Flat \\
Limited \\
\end{tabular} & \begin{tabular}{|l|} 
Static \\
Dynamic \\
\end{tabular} & Yes & $\iota \delta$ & Active & Yes & No & $G$ & No & No & - & $\mathrm{No}$ & No \\
\hline 95 & $\mathrm{~N}$ & $\mathrm{No}$ & Maintain & $\begin{array}{l}\text { Comp } \\
\text { Run }\end{array}$ & Relation & No & \begin{tabular}{|l|} 
Flat \\
Limited \\
\end{tabular} & Static & Yes & $\iota \delta$ & Active & Yes & No & S & No & No & - & No & No \\
\hline 75 & $\mathrm{~N}$ & Yes & Check & Run & Logic & Yes & Limited & Static & Yes & $\iota \delta$ & SLD & Yes & No & S & Yes & No & $1-6,9$ & Yes & Yes \\
\hline [1] & $\mathrm{O}$ & No & Maintain & Run & Logic & Yes & Limited & Static & Yes & $\iota \delta$ & - & Yes & No & S & Yes & No & - & No & No \\
\hline 96 & $\mathrm{~N}$ & No & Maintain & Comp & Relation & No & Limited & Static & Yes & $\iota \delta$ & $\begin{array}{l}\text { Predef } \\
\text { Program }\end{array}$ & No & No & $G$ & No & No & - & No & No \\
\hline 53 & $\mathrm{~N}$ & No & Maintain & Comp & Logic & Yes & Limited & Static & Yes & $\iota \delta$ & - & No & No & S & No & No & - & $\mathrm{No}$ & No \\
\hline [26] & $\mathrm{N}$ & No & Maintain & $\begin{array}{l}\text { Comp. } \\
\text { Run }\end{array}$ & Relation & Yes & Limited & \begin{tabular}{|l|} 
Static \\
Dynamic \\
\end{tabular} & Yes & $\iota \delta$ & - & Yes & No & S & Yes & No & - & \begin{tabular}{|l|} 
Not \\
proved
\end{tabular} & $\begin{array}{l}\text { Not } \\
\text { proved }\end{array}$ \\
\hline 40 & $\mathrm{H}$ & Yes & Check & Run & Logic & Yes & Yes & Static & Yes & $\iota \delta$ & \begin{tabular}{|l|} 
Predef \\
Programs \\
\end{tabular} & Yes & No & S & Yes & No & $1-6,7$ & Yes & \begin{tabular}{|l} 
Not \\
proved
\end{tabular} \\
\hline 55 & $\mathrm{O}$ & Yes & Check & Run & Relation & Yes & Limited & Static & $\mathrm{No}$ & $\iota \delta$ & Unfold & Yes & No & S & Yes & No & $1-6,9$ & Yes & Yes \\
\hline 10 & $\mathrm{O}$ & Yes & Check & Run & Relation & Yes & Limited & Static & No & $\iota \delta$ & Unfold & Yes & No & S & Yes & No & $1-6,9$ & Yes & Yes \\
\hline 43 & $\mathrm{~N}$ & Yes & Check & Run & Logic & Yes & Yes & Static & Yes & $\iota \delta$ & SLDNF & Yes & No & S & Yes & No & $1-6,9$ & Yes & Yes \\
\hline 91 & $\mathrm{~N}$ & No & Maintain & Run & Logic & Yes & Limited & Static & Yes & $\iota \delta$ & Predef & Yes & No & S & Yes & No & $1-6,7$ & Yes & $\begin{array}{l}\text { Not } \\
\text { proved }\end{array}$ \\
\hline 59 & $\mathrm{O}$ & No & Maintain & Comp & Relation & Yes & Limited & Static & Yes & $\iota \delta$ & - & Yes & No & S & Yes & No & - & No & No \\
\hline
\end{tabular}




\begin{tabular}{|c|c|c|c|c|c|c|c|c|c|c|c|c|c|c|c|c|c|c|c|}
\hline \multirow[b]{2}{*}{ Method } & \multicolumn{4}{|c|}{ Problem } & \multicolumn{4}{|c|}{ Database schema } & \multicolumn{2}{|c|}{ Update req. } & \multicolumn{3}{|c|}{ Mechanism } & \multicolumn{3}{|c|}{ Update Change } & \multicolumn{3}{|c|}{ Solutions } \\
\hline & Type & \begin{tabular}{|l|} 
View \\
Update
\end{tabular} & \begin{tabular}{|l|}
$\mathrm{IC}$ \\
Enforce. \\
\end{tabular} & \begin{tabular}{|l|} 
Run/ \\
Comp.
\end{tabular} & \begin{tabular}{|l|} 
Def. \\
Lang.
\end{tabular} & View & \begin{tabular}{|l|} 
IC \\
def.
\end{tabular} & \begin{tabular}{|l} 
Kind of \\
IC
\end{tabular} & Mul. & \begin{tabular}{|l|} 
Update \\
Operat.
\end{tabular} & \begin{tabular}{|l|}
$\begin{array}{l}\text { Tech- } \\
\text { nique }\end{array}$ \\
\end{tabular} & \begin{tabular}{|l|} 
Base \\
Facts \\
\end{tabular} & $\begin{array}{l}\text { User } \\
\text { Part. }\end{array}$ & Type & $\begin{array}{l}\text { Base } \\
\text { facts }\end{array}$ & \begin{tabular}{|l} 
View \\
def.
\end{tabular} & Axiom & Sound. & Complete. \\
\hline 92 & $\mathrm{~N}$ & Yes & Check & Run & Logic & No & Limited & Static & Yes & $\iota \delta$ & SLDNF & Yes & No & $\mathrm{S}$ & Yes & No & $1-6,9$ & Yes & Yes \\
\hline [44] & $\mathrm{N}$ & Yes & Check & Run & Logic & Yes & Yes & Static & No & $\iota \delta$ & SLDNF & No & No & $\mathrm{S}$ & Yes & No & $1-6,9$ & Not & $\begin{array}{l}\text { No } \\
\text { proved }\end{array}$ \\
\hline 80 & $\mathrm{~N}$ & Yes & \begin{tabular}{|l|} 
Check \\
Maintain
\end{tabular} & Run & Logic & Yes & Yes & Static & No & $\iota \delta \chi$ & SLD & Yes & No & S & Yes & No & - & No & No \\
\hline [17. & $\mathrm{N}$ & Yes & \begin{tabular}{|l|} 
Check \\
Maintain
\end{tabular} & \begin{tabular}{|l|} 
Run \\
Comp
\end{tabular} & Logic & Yes & Yes & Static & No & $\iota \delta \chi$ & SLD & Yes & No & SS & Yes & No & $1-6,7$ & Yes & $\begin{array}{l}\text { Not } \\
\text { proved }\end{array}$ \\
\hline [27] & $\mathrm{N}$ & Yes & \begin{tabular}{|l|} 
Check \\
Maintain
\end{tabular} & Run & Logic & Yes & Yes & \begin{tabular}{|l|} 
Static \\
Dynamic
\end{tabular} & Yes & $\iota \delta \chi$ & Predef & Yes & No & S & Yes & No & $1-6,9$ & Yes & Yes \\
\hline 21 & $\mathrm{~N}$ & Yes & Check & Comp & Logic & Yes & Yes & Dynamic & Yes & $\iota \delta$ & $\begin{array}{l}\text { Predef } \\
\text { Programs } \\
\end{array}$ & Yes & No & $\mathrm{S}$ & Yes & No & - & \begin{tabular}{|l|} 
Not \\
Proved
\end{tabular} & $\begin{array}{l}\text { No } \\
\text { proved }\end{array}$ \\
\hline 28 & $\mathrm{~N}$ & Yes & \begin{tabular}{|l|} 
Check \\
Maintain
\end{tabular} & Run & Logic & Yes & Yes & \begin{tabular}{|l|} 
Static \\
Dynamic
\end{tabular} & Yes & $\iota \delta \chi$ & Predef & Yes & No & $\mathrm{S}$ & Yes & No & $1-6,9$ & Yes & Yes \\
\hline 30 & $\mathrm{~N}$ & No & Maintain & Comp & Logic & Yes & No & - & Yes & $\iota \delta$ & - & Yes & No & S & Yes & No & - & No & No \\
\hline 106 & $\mathrm{~N}$ & No & Maintain & Run & Relation & Yes & No & - & Yes & $\iota \delta \chi$ & Unfold & Yes & No & SS & No & No & - & \begin{tabular}{|l|} 
Not \\
proved
\end{tabular} & \begin{tabular}{|l}
$\begin{array}{l}\text { Not } \\
\text { proved }\end{array}$ \\
\end{tabular} \\
\hline$[56$ & $\mathrm{O}$ & No & Maintain & \begin{tabular}{|l|} 
Comp. \\
Run
\end{tabular} & Logic & Yes & No & - & Yes & $\iota \delta$ & - & Yes & No & $G$ & No & No & - & Yes & $\begin{array}{l}\text { Not } \\
\text { proved }\end{array}$ \\
\hline 11. & S & Yes & Check & Run & Logic & Yes & \begin{tabular}{|l|} 
Flat \\
Limited \\
\end{tabular} & Static & Yes & $\iota \delta$ & SLDNF & Yes & No & S & Yes & No & $1-6,9$ & Yes & \begin{tabular}{|l}
$\begin{array}{l}\text { Not } \\
\text { proved }\end{array}$ \\
\end{tabular} \\
\hline 441 & $\mathrm{N}$ & Yes & Check & Run & Logic & Yes & Yes & Static & Yes & $\iota \delta$ & - & Yes & No & S & Yes & No & - & No & No \\
\hline 23 & $\mathrm{O}$ & No & Maintain & Run & Relation & Yes & No & Static & Yes & $\iota \delta$ & SLD & Yes & Yes & $G$ & No & No & - & $\begin{array}{l}\text { Not } \\
\text { proved }\end{array}$ & \begin{tabular}{|l} 
Not \\
proved
\end{tabular} \\
\hline 12 & $\mathrm{O}$ & No & Maintain & Comp & Relation & Yes & No & Static & Yes & $\iota \delta \chi$ & - & Yes & No & $\mathrm{SS}$ & Yes & No & - & No & No \\
\hline [3] & $\mathrm{O}$ & No & Maintain & $\begin{array}{l}\text { Comp. } \\
\text { Run }\end{array}$ & Relation & No & Limited & $\begin{array}{l}\text { Static } \\
\text { Dynamic }\end{array}$ & Yes & $\iota \delta$ & - & Yes & No & $G$ & Yes & No & - & No & No \\
\hline [78 & $\mathrm{N}$ & No & Maintain & Comp & Relation & No & Yes & Static & Yes & $\iota \delta \chi$ & Unfold & No & Yes & SS & No & No & - & No & No \\
\hline 93 & $\mathrm{~N}$ & No & Check & Comp & Logic & No & Yes & Static & Yes & $\iota \delta$ & Active & Yes & No & $G$ & Yes & No & - & No & No \\
\hline 36 & $\mathrm{~N}$ & Yes & Check & Run & Logic & Yes & Yes & Static & Yes & $\iota \delta$ & SLD & Yes & No & S & Yes & No & $1-6,9$ & Yes & Yes \\
\hline
\end{tabular}




\section{References}

1. Agrawal, S., et al. (2008). Automated selection of materialized views and indexes in SQL databases, Intl. Conference on Very Large Data Bases (VLDB), 496-515.

2. Alchourron, C.E., et al.(1985). On the logic of theory change: Partial meet contraction and revision functions. Journal of Symbolic Logic 50, 510 - 530.

3. Alexandre, L., \& Coelho, J. (2011). Filtering XML content for publication and presentation on the web, ICDIM, 85-89.

4. Aliseda, A. (2006). Abductive Resoning Logic Investigations into Discovery and Explanation. Springer book series Vol. 330.

5. Amgoud, L. \& Vesic, S. (2011). A new approach for preference-based argumentation frameworks, Ann. Math. Artif. Intell., 63(2), 149-183.

6. Aravindan, C.,\& Dung, P.M.(1994). Belief Dynamics, Abduction, and Database. JELIA, 66-85.

7. Aravindan C.(1995), Dynamics of Belief: Epistmology, Abduction and Database Update. Phd Thesis, AIT.

8. Aravindan. C., \& Baumgartner. P. (1997). A Rational and Efficient Algorithm for View Deletion in Databases. ILPS, 165-179.

9. Baumgartner, P., et al. (1997). Semantically Guided Theorem Proving for Diagnosis Applications. IJCAI 1, 460-465.

10. Bauer, A., \& Lehne, W. (2003). On solving the view selection problem in distributed data warehouse architectures. SSDBM, 43-57.

11. Behrend, A.,\& Manthey,R. (2008). A Transformation-Based Approach to View Updating in Stratifiable Deductive Databases. FoIKS, 253-271.

12. Bellahsene, Z., et al., (2010). A cooperative approach to view selection and placement in P2P systems. OTM, 515-522. 2010.

13. Bentahar, J., et al. (2008). Agent-based communities of web services: an argumentation-driven approach, Service Oriented Computing and Applications, 2(4), 219-238.

14. Bentahar, J., et al. (2010). Using argumentation to model and deploy agent-based B2B applications, Knowl.-Based Syst., 23(7), 677-692.

15. Bentahar, J., et al. (2010). A taxonomy of argumentation models used for knowledge representation, Artif. Intell. Rev, 33(3), 211-259.

16. Bessant, B., et al.(1998). Combining Nonmonotonic Reasoning and Belief Revision: A Practical Approach. AIMSA, 115-128.

17. Braga, D., et al. (2006). Efficient Integrity Checking over XML Documents, EDBT Workshops, 206-219.

18. Biskup, J. (2012). Inference-usability confinement by maintaining inference-proof views of an information system. IJCSE. 7 (1), 17-37.

19. Caroprese, L., et al.(2012). The View-Update Problem for Indefinite Databases. JELIA.

20. Calvanese, D., et al. (2012). View-based query answering in Description Logics Semantics and complexity. J. Comput. Syst. Sci 78(1), 26-46.

21. Caroprese, L., et al. (2007). View Updating Through Active Integrity Constraints, ICLP, 430-431.

22. Ceri, S., et al. (1994). Automatic Generation of Production Rules for Integrity Maintenance, ACM Transactions on Database Systems 19(3), 367-422.

23. Chaves, L.W.F., et al. (2009). Towards materialized view selection for distributed databases, EDBT, 1088-1109. 
24. Chen, H., \& Liao, H. (2010). A Comparative Study of View Update Problem. DSDE, 83-89.

25. Chen, I.A., et al. (1995). An Execution Model for Limited Ambiguity Rules and Its Application to Derived Data Update, ACM Transactions on Database Systems 20, (4), 365-413.

26. Chirkova, R. (2002). The view-selection problem has an exponential-time lower bound for conjunctive queries and views. ACM Symposium on Principles of Database Systems (PODS), 159-168.

27. Christiansen, H., \& Martinenghi, D. (2006). On Simplification of Database Integrity Constraints, Fundam. Inform 71(4), 371-417.

28. Christiansen, H., \& Rekouts, M. (2007). Integrity Checking and Maintenance with Active Rules in XML Databases, BNCOD Workshops, 59-67.

29. Christiansen, H., \& Dahl,V. (2009). Abductive Logic Grammars. WoLLIC, 170181.

30. Coelho, J., \& Florido, M. (2007). Type-Based Static and Dynamic Website Verification, ICIW, 32 .

31. Cong, G., et al. (2012). On the Complexity of View Update Analysis and Its Application to Annotation Propagation. IEEE Trans. Knowl. Data Eng.24(3), 506-519.

32. Console, L., et al. (1995). The Role of Abduction in Database View Updating, Journal of Intelligent Information Systems 4, 261-280.

33. Decker, H., (1997). One Abductive Logic Programming Procedure for two kind of Updates, Proc. Workshop DINAMICS'97 at Int. Logic Programming Symposium.

34. Delhibabu, R., \& Lakemeyer, G. (2013). A Rational and Efficient Algorithm for View Revision in Databases. Applied Mathematics $\&$ Information Sciences 7(3).

35. Delhibabu, R. (2014). An Abductive Framework for Knowledge Base Dynamics. Applied Mathematics \& Information Sciences (accepted).

36. Delhibabu, R., \& Behrend, A. (2014). A New Rational Algorithm for View Updating in Relational Databases. Applied Intelligence (accepted).

37. Delgrande, J.P, \& Peppas, P. (2011). Revising Horn Theories. IJCAI, 839-844.

38. Dhote, C. A., \& Ali, M. S. (2009). Materialized View Selection in Data Warehousing: A Survey. Journal of Applied Sciences, 401-414.

39. Dong, G. \& Topor, R. W. (1992). Incremental evaluation of datalog queries. In Database Theory - ICDT, volume 646, 282-296.

40. Domínguez, E., et al. (2002). Integrity Constraint Enforcement by Means of Trigger Templates, ADVIS, 54-64.

41. Domínguez, E., et al. (2008). Model-Driven, View-Based Evolution of Relational Databases, DEXA, 822-836.

42. Eiter, T., \& Makino,K. (2007). On computing all abductive explanations from a propositional Horn theory. J. ACM 54 (5).

43. Farré, C., et al. (2003). Handling Existential Derived Predicates in View Updating, ICLP, 148-162.

44. Farré, C., et al. (2004). A New Approach for Checking Schema Validation Properties. DEXA, 77-86.

45. Falappa, M.A., et al.(2012). Prioritized and Non-prioritized Multiple Change on Belief Bases. J. Philosophical Logic 41 (1), 77-113.

46. Falappa, M, A., et al. (2013). Stratified Belief Bases Revision with Argumentative Inference, J. Philosophical Logic, 42(1), 161-193.

47. Ferme, E.L., \& Hansson, S.O. (2011). AGM 25 Years - Twenty-Five Years of Research in Belief Change. J. Philosophical Logic 40 (2),295-331. 
48. Fraternali, P., \& Paraboschi, S. (1993). A Review of Repairing Techniques for Integrity Maintenance, RIDS, 333-346.

49. Gertz, M. (1994). Specifying Reactive Integrity Control for Active Databases, RIDE, 62-70.

50. Guessoum, A., \& Lloyd, J.W. (1990). Updating Knowledge Bases, New Generation Computing Vol, 8 (1), 71-89.

51. Gupta, A., et al. (1992). Counting solutions to the view maintenance problem. In Workshop on Deductive Databases, JICSLP, 185-194.

52. Gupta, A., et al. (1993). Maintaining views incrementally. ACM SIGMOD, 157-176.

53. Halevy, A. Y. (2001). Answering queries using views: A survey. $V L D B, \mathbf{1 0}(4), 270 \ddot{£} \cdot 1 \ddot{\jmath} £ \cdot 74$.

54. Hansson, S.O. (1997).A Textbook of Belief Dynamics. Kluwer Academic Publishers, Dordrecht.

55. Hegner, S. J. (2002). Uniqueness of Update Strategies for Database Views. FoIKS, 230-249.

56. Hegner, S. J. (2007). A Model of Database Components and their Interconnection Based upon Communicating Views. EJC, 79-99.

57. Heras, S., et al. (2013). Research opportunities for argumentation in social networks, Artif. Intell. Rev., 39(1), 39-62.

58. Herzig, A. \& Rifi,O. (1999). Propositional Belief Base Update and Minimal Change. Artif. Intell. 115(1), 107-138.

59. Horng, J. T., et al. (2003). Applying evolutionary algorithms to materialized view selection in a data warehouse, Soft Comput, 7(8),574-591.

60. Jackson, E. K. \& Schulte, W. (2008). Model Generation for Horn Logic with Stratified Negation. FORTE.

61. Kakas, A.C., \& Mancarella,P. (1990). Database Updates Through Abduction, VLDB Conference, 650-661.

62. Keller, A. (1985). Updating Relational Databases Through Views. Phd Thesis.

63. Kogalovsky, M.R. (2012). Ontology-based data access systems. Programming and Computer Software 38(4), 167-182.

64. Konieczny, S. (2011). Dynamics of Beliefs. SUM, 61-74.

65. Küchenhoff. (1991). On the efficient computation of the difference between consecutive database states. DOOD, 478-492.

66. Lakemeyer, G. (1995). A Logical Account of Relevance. IJCAI (1), 853-861.

67. Langlois, M., et al. (2008). Horn Complements: Towards Horn-to-Horn Belief Revision. $A A A I, 466-471$.

68. Laurent, D., et al. (1998). Updating Intensional Predicates in Deductive Databases. Data Knowl. Eng. 26(1), 37-70.

69. Lee, M., \& Hammer, J. (2001). Speeding up materialized view selection in data warehouses using a randomized algorithm, Int. J. Cooperative Inf. Syst., 10(3), 327-343.

70. Liberatore, P. (1997). The Complexity of Belief Update (Extended in 2003). IJCAI vol. 1, pp. 68-73. 68-73.

71. Liberatore, P., \& Schaerf, M. (2004). The Compactness of Belief Revision and Update Operators. Fundam. Inform. 62(3-4), 377-393.

72. Lobo, J., \& Trajcevski, G. (1997). Minimal and Consistent Evolution of Knowledge Bases, Journal of Applied Non-Classical Logics 7(1), pp. 117-146..

73. Li, X. (2010). Materialized View Selection: A Survey, IGI book chapter, View Management Techniques and Their Application to Data Stream Management, DOI: 10.4018/978-1-60566-816-1.ch005. 
74. Lu, J., et al. (1995). Efficient maintenance of materialized mediated views. $A C M$ SIGMOD, 340-351.

75. Lu, W. (1999). View Updates in Disjunctive Deductive Databases Based on SLDResolution. KRDB, 31-35.

76. Maabout, S. (1998). Maintaining and Restoring Database Consistency with Update Rules, Workshop DYNAMICS, JICSLP.

77. Makinson, D. (1997). Screened Revision, Theoria 63, 14-23.

78. Mami, I., et al. (2011). Modeling view selection as a constraint satisfaction problem. $D E X A$, vol. $6861,396-410$.

79. Mami, I., \& Bellahsene. Z. (2012). A survey of view selection methods, SIGMOD Record 41 (1), 20-29.

80. Martinenghi, D., \& Christiansen, H. (2005). Efficient Integrity Checking for Databases with Recursive Views. ADBIS, vol. 3631, 109-124.

81. Martinez, M, V., et al. (2013). A General Framework for Reasoning On Inconsistency, Springer Briefs in Computer Science, Vol. 7, 1-45.

82. Mayol, E., \& Teniente, E. (1993). Incorporating Modification Requests in Updating Consistent Knowledge Bases, Fourth Int. Works. on the Deductive Approach to Information Systems and Databases, 275-300.

83. Mayol, E., \& Teniente, E. (1999). A Survey of Current Methods for Integrity Constraint Maintenance and View Updating. ER (Workshops), 62-73.

84. Moerkotte, G., \& Lockemann, P.C.(1991). Reactive Consistency Control in Deductive Databases, ACM Transactions on Database Systems, 16 (4), 670-702.

85. Mota-Herranz, L., et al. (2000). Transaction Trees for Knowledge Revision, FQAS, 182-191.

86. Nayak, A., et al. (2006). Forgetting and Knowledge Update. Australian Conference on Artificial Intelligence, 131-140.

87. Nayak, A. (2011). Is Revision a Special Kind of Update? Australasian Conference on Artificial Intelligence, 432-441.

88. Nebel, B. (1998). How Hard is it to Revise a Belief Base? Handbook of Defeasible Reasoning and Uncertainty Management Systems, 77-145.

89. Papini, O.(2000). Knowledge-base revision. The Knowledge Engineering Review 15(4), 339 - 370.

90. Qi, G., \& Yang, F. (2008). A Survey of Revision Approaches in Description Logics. Description Logics.

91. Saha, D., \& Ramakrishnan, C. R. (2003). Incremental Evaluation of Tabled Logic Programs. ICLP, 392-406.

92. Sakama, C., \& Inoue, K. (2003). An abductive framework for computing knowledge base updates. TPLP 3(6), 671-713.

93. Salman, M., et al. (2012). Database Integrity Mechanism between OLTP and Offline Data, ACIIDS, vol. 2, 371-380.

94. Schewe, K.D. (1996). Tailoring Consistent Specializations as a Natural Approach to Consistency Enforcement, 6th Int. Workshop on Foundations of Models and Languages for Data and Objects: Integrity in Databases.

95. Schewe, K. D. (1998). Consistency Enforcement in Entity-Relationship and Object Oriented Models, Data \& Knowledge Eng 28(1),121-140.

96. Schewe, K. D. (2000). Controlled Automation of Consistency Enforcement, ASE, 265-268.

97. Schulte, O. (1999). Minimal Belief Change and Pareto-Optimality. Australian Joint Conference on Artificial Intelligence, 144-155.

98. Segerberg, K. (1998). Irrevocable Belief Revision in Dynamic Doxastic Logic. Notre Dame Journal of Formal Logic 39(3), 287-306. 
99. Staudt, M., \& and Jarke. M. (1996). Incremental maintenance of externally materialized views. VLDB Journal, $75 \ddot{i} £ \cdot 1 \ddot{1} £ \cdot 7$.

100. Teniente, E., \& Olive, A. (1995). Updating Knowledge Bases while Maintaining their Consistency, The VLDB Journal, 4(2), 193-241.

101. Urpí \& Olivé, A. (1992). A method for change computation in deductive databases. $V L D B, 225 \mathrm{ï} \cdot 1 \ddot{1} £ \cdot 77$.

102. Wrobel, S. (1995). First order Theory Refinement. IOS Frontier in AI and Application Series.

103. Wüthrich, B. (1993). On Updates and Inconsistency Repairing in Knowledge Bases, ICDE, 608-615.

104. Yang, J., et al. (1997). Algorithms for materialized view design in data warehousing environment, $V L D B, 136-145$.

105. Zhang, C., \& Yang, Y. (1999). Genetic algorithm for materialized view selection in data warehouse environments, DaWaK, vol. 1676, 116-125. 1999.

106. Zhou, J., et al. (2007). Dynamic materialized views. ICDE, 526-535. 